\title{
Viewing determinants as nonintersecting lattice paths yields classical determinantal identities bijectively
}

\author{
Markus Fulmek* \\ Fakultät für Mathematik \\ Nordbergstraße 15, A-1090 Wien, Austria \\ Markus.Fulmek@univie.ac.at
}

Submitted: Feb 16, 2012; Accepted: Jul 12, 2012; Published: Aug 16, 2012

Mathematics Subject Classifications: 05A19, 05E05

\begin{abstract}
In this paper, we show how general determinants may be viewed as generating functions of nonintersecting lattice paths, using the Lindström-Gessel-Viennotmethod and the Jacobi-Trudi identity together with elementary observations. After some preparations, this point of view provides "graphical proofs" for classical determinantal identities like the Cauchy-Binet formula, Dodgson's condensation formula, the Plücker relations, Laplace's expansion and Turnbull's identity. Also, a determinantal identity generalizing Dodgson's condensation formula is presented, which might be new.
\end{abstract}

\section{Introduction}

In [6], a combinatorial proof was given for two Schur function identities, which were presented in [14] and in [15]. This combinatorial proof was shown to apply to a class of Schur function identities [6, Lemma 16], and was used to prove bijectively Dodgson's condensation formula and the Plücker relations but was not paid further attention. Recently, members of this class of Schur function identities received some interest [10]. The close connection of the result $[10,(3.3)]$ to the work in [6] was already explained ad hoc in [5], but we take this opportunity to make obvious the much wider range of applicability of this idea, which amounts to the following statement:

$m \times m$-determinants may be viewed as (generating functions of) $m$-tuples of nonintersecting lattice paths in $\mathbb{Z}^{2}$ with starting points on some fixed horizontal line $y=\alpha$ and ending points on some fixed horizontal line $y=\omega>\alpha$, where

\footnotetext{
*Research supported by the National Research Network "Analytic Combinatorics and Probabilistic Number Theory", funded by the Austrian Science Foundation.
} 
- the rows of the determinants (in the usual order: top to bottom) correspond to the starting points of the lattice paths (ordered from right to left),

- and the columns of the determinants (in the usual order: left to right) correspond to the ending points of the lattice paths (ordered from right to left).

This statement, of course, has to be explained properly, and we shall give several concrete examples to illustrate its meaning. The combinatorial constructions are best conceived by pictures, so we give a lot of illustrations.

This paper is organized as follows:

In Section 2, we present basic background information regarding symmetric functions, partitions, Young tableaux and (skew) Schur functions.

In Section 3, we present the weight-preserving bijection between Young tableaux and nonintersecting lattice paths, which (in some sense) exposes determinants as generating functions of nonintersecting lattice paths. We illustrate this view by giving a "graphical proof" of the Cauchy-Binet formula. (I am grateful to the anonymous referee for drawing my attention to the paper [1], which gives a similar proof in an easily comprehensible exposition.)

In Section 4, we present the central bijective construction (recolouring of bicoloured trails in the overlays of families of nonintersecting lattice paths, which correspond to some product of skew Schur functions) and indicate how this construction gives rise to Schur function identities.

In Section 5, we present several examples: We prove a generalization of Dodgson's condensation rule which might be new (Theorem 2) and give "graphical proofs" for the Plücker relations (and its generalization [10, (3.3)]), for (a generalization of) Laplace's expansion, and for Turnbull's identity.

\section{Basic definitions}

The notation $|x|$ has three different meanings in our presentation, depending on the type of object $x$ (the meaning should always be clear from the context):

- if $x$ is a set, then $|x|$ denotes the cardinality of $x$,

- if $x$ is a matrix, then $|x|$ denotes the determinant of $x$,

- if $x$ is a partition or shape, then $|x|$ denotes the sum of parts of $x$ (to be explained below).

In the following, we shall briefly recall basic concepts and facts needed for our presentation. (More information can be found, e.g., in [19].) 


\subsection{The ring of symmetric functions}

Consider the ring $\mathbb{Z}\left[x_{1}, x_{2}, \ldots, x_{N}\right]$ of polynomials in $N$ independent (commuting) variables $\mathbf{x}:=\left(x_{1}, x_{2}, \ldots, x_{N}\right)$ with integer coefficients. The degree of a monomial $x_{1}^{k_{1}}$. $x_{2}^{k_{2}} \cdots x_{N}^{k_{N}}$ is the sum $k_{1}+k_{2}+\cdots+k_{N}$, and a polynomial $p$ is called homogeneous of degree $k$ if all monomials of $p$ have the same degree $k$.

The symmetric group $\mathfrak{S}_{N}$ acts on this ring by permuting the variables, and a polynomial is symmetric if it is invariant under this action. The set of all symmetric polynomials forms a subring $\Lambda_{n} \subseteq \mathbb{Z}\left[x_{1}, x_{2}, \ldots, x_{N}\right]$ which is graded, i.e.,

$$
\Lambda_{N}=\bigoplus_{k \geqslant 0} \Lambda_{N}^{k}
$$

where $\Lambda_{N}^{k}$ consists of the homogeneous symmetric polynomials of degree $k$, together with the zero polynomial.

For each $r \in \mathbb{Z}$, the complete homogeneous symmetric function $h_{r}(\mathbf{x})$ is the sum of all monomials of degree $r$. In particular, $h_{0}(\mathbf{x})=1$ and, by convention, $h_{r}(\mathbf{x})=0$ for $r<0$.

For example, if $N=3$, then $\mathbf{x}=\left(x_{1}, x_{2}, x_{3}\right)$ and

$$
h_{2}\left(x_{1}, x_{2}, x_{3}\right)=x_{1}^{2}+x_{2}^{2}+x_{3}^{2}+x_{1} x_{2}+x_{1} x_{2}+x_{2} x_{3} .
$$

The following fact is well-known (see, e.g., [19, (2.8)]):

Proposition 1. The set of complete homogeneous symmetric functions

$$
\left\{h_{0}(\mathbf{x}), h_{1}(\mathbf{x}), \ldots, h_{N}(\mathbf{x})\right\}
$$

is algebraically independent, i.e., $p \equiv 0$ is the only polynomial such that

$$
p\left(h_{0}(\mathbf{x}), h_{1}(\mathbf{x}), \ldots, h_{N}(\mathbf{x})\right) \equiv 0 .
$$

Moreover, the set

$$
\left\{h_{i}\left(x_{1}, \ldots, x_{N}\right): i=0,1,2, \ldots, N\right\} \cup\left\{h_{i}\left(x_{N+1}, \ldots, x_{2 N}\right): i=1,2, \ldots, N\right\}
$$

is also algebraically independent.

\subsection{Schur functions}

Recall the following standard definitions: An infinite weakly decreasing series of nonnegative integers $\lambda=\left(\lambda_{i}\right)_{i=1}^{\infty}$, where only finitely many elements are positive, is called a partition. The largest index $i$ for which $\lambda_{i}>0$ is called the length of the partition $\lambda$ and is denoted by $\ell(\lambda)$. The sum of the non-zero parts $\lambda_{1}+\lambda_{2}+\cdots+\lambda_{\ell(\lambda)}$ of $\lambda$ is denoted by $|\lambda|$. In most cases we shall omit the trailing zeroes, i.e., for $\ell(\lambda)=r$ we simply write $\lambda=\left(\lambda_{1}, \lambda_{2}, \ldots, \lambda_{r}\right)$, where $\lambda_{1} \geqslant \lambda_{2} \geqslant \ldots \geqslant \lambda_{r}>0$.

For example, $\lambda=(7,4,4,3,1,1,1)$ is a partition of length $\ell(\lambda)=7$ with $|\lambda|=21$. 
Figure 1: Illustration: Ferrers diagram $F_{\lambda}$ of the partition $\lambda=(7,4,4,3,1,1,1)$.

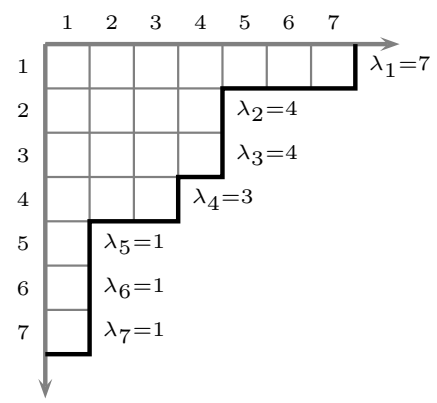

The Ferrers diagram $F_{\lambda}$ of $\lambda$ is an array of cells with $\ell(\lambda)$ left-justified rows and $\lambda_{i}$ cells in row $i$. For an illustration, see Figure 1.

For our purposes, it is convenient to generalize this definition: By a semipartition we understand an infinite weakly decreasing series of integers $\left(\lambda_{i}\right)_{i=1}^{\infty}$, where

$$
\lambda_{\infty}:=\lim _{n \rightarrow \infty} \lambda_{n}>-\infty .
$$

The length of semipartition $\lambda$ is the largest integer $m$ with $\lambda_{m}>\lambda_{\infty}$, which we denote again by $\ell(\lambda)$ : Note that every partition $\mu$ is a semipartition with $\mu_{\infty}=0$.

Clearly, the set of semipartitions is closed under component-wise addition

$$
\lambda+\mu=\left(\lambda_{i}\right)_{i=1}^{\infty}+\left(\mu_{i}\right)_{i=1}^{\infty}:=\left(\lambda_{i}+\mu_{i}\right)_{i=1}^{\infty} .
$$

For $z \in \mathbb{Z}$, denote by $(\bar{z})$ the semipartition

$$
(\bar{z}):=(z)_{i=1}^{\infty}
$$

For $m, z \in \mathbb{N}$, denote by $\left(z^{\lfloor m\rceil}\right)$ the partition

$$
\left(z^{\lfloor m\rceil}\right):=(\underbrace{z, z, \ldots, z}_{m \text { times }}, 0,0, \ldots) .
$$

If two semipartitions $\lambda, \mu$ satisfy

- $\mu_{i} \leqslant \lambda_{i}$ for all $i=1,2, \ldots$,

- and $\mu_{\infty}=\lambda_{\infty}$,

then we denote this by $\mu \unlhd \lambda$ and introduce the symbol $\lambda / \mu$, which we call a shape. The length of the shape $\lambda / \mu$ is defined by $\ell(\lambda / \mu):=\ell(\lambda)$, and the (terminating!) sum $\sum_{i=1}^{\infty}\left(\lambda_{i}-\mu_{i}\right)$ is denoted by $|\lambda / \mu|$. Note that we may view partition $\lambda$ as the shape $\lambda /(\overline{0})$ 
Figure 2: Illustration: Ferrers diagram of skew shape.

Ferrers diagram $F_{\lambda / \mu}$ of the shape $\lambda / \mu=(7,4,4,3,1,1,1) /(3,2,2,1)$. Note that the same Ferrers diagram would arise for the shapes $(\lambda+(\bar{z})) /(\mu+(\bar{z}))$ for arbitrary $z \in \mathbb{Z}$, and for shapes $\left(\lambda+\left(z^{\lfloor 7\rceil}\right)\right) /\left(\mu+\left(z^{\lfloor 7\rceil}\right)\right)$, for $z \in \mathbb{N}$.

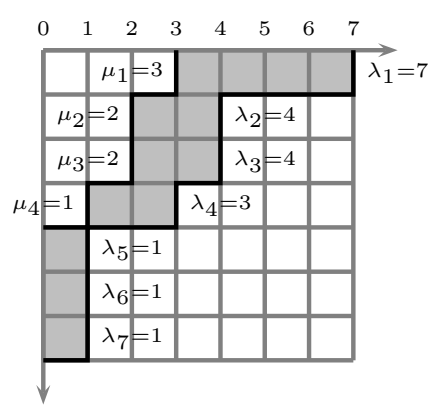

The Ferrers diagram $F_{\lambda / \mu}$ of shape $\lambda / \mu$ is an array of cells with $\ell(\lambda / \mu)$ left-justified rows and $\left(\lambda_{i}-\mu_{i}\right)$ cells in row $i$, where the first $\mu_{i}$ cells in row $i$ are missing, see Figure 2 for an illustration.

Note that for arbitrary $z \in \mathbb{Z}$, the Ferrers diagram $F_{\lambda / \mu}$ with $\ell(\lambda / \mu)=m$ is congruent to

$$
\begin{aligned}
& F_{\lambda / \mu} \simeq F_{\lambda+(\bar{z}) / \mu+(\bar{z})} \text { for arbitrary } z \in \mathbb{Z}, \\
& F_{\lambda / \mu} \simeq F_{\lambda+\left(z^{\lfloor m\rceil}\right) / \mu+\left(z^{\lfloor m\rceil}\right)} \text { for } z>0 \in \mathbb{Z} .
\end{aligned}
$$

In particular, for some partition $\lambda$ we have $F_{\lambda} \simeq F_{\lambda+(\bar{z}) /(\bar{z})}$ for arbitrary $z \in \mathbb{Z}$.

Schur functions, which are irreducible general linear characters, can be defined as follows [23]. Let $\lambda$ be a partition and let $\left\{x_{1}, \ldots, x_{N}\right\}$ be a set of independent variables. Then the Schur function $s_{\lambda}\left(x_{1}, \ldots, x_{N}\right)$ indexed by $\lambda$ is defined as the quotient of determinants (see $[19,(3.1)])$

$$
s_{\lambda}\left(x_{1}, \ldots, x_{N}\right):=\frac{\left|x_{i}^{\lambda_{j}+N-j}\right|_{i, j=1}^{N}}{\left|x_{i}^{N-j}\right|_{i, j=1}^{N}} .
$$

It is easy to see that $s_{\lambda}$ is a symmetric function, which is homogeneous of degree $|\lambda|$. The Jacobi-Trudi identity (first obtained by Jacobi [12] and simplified by Trudi [25], see $[19,(3.4)])$ states that the Schur function $s_{\lambda}$ equals the following determinant of complete homogeneous symmetric functions:

$$
s_{\lambda}=\left|h_{\lambda_{j}-j+i}\right|_{i, j=1}^{m},
$$

where $m=\ell(\lambda)$. Here, we introduced in passing the shorthand notations $s_{\lambda}$ and $h_{r}$ for $s_{\lambda}\left(x_{1}, \ldots, x_{N}\right)$ and $h_{r}\left(x_{1}, \ldots, x_{N}\right)$, respectively. 
Figure 3: Illustration: An 8-semistandard Young tableau $T$ of shape $\lambda=(7,4,4,3,1,1,1)$ and its weight $\omega(T)$.

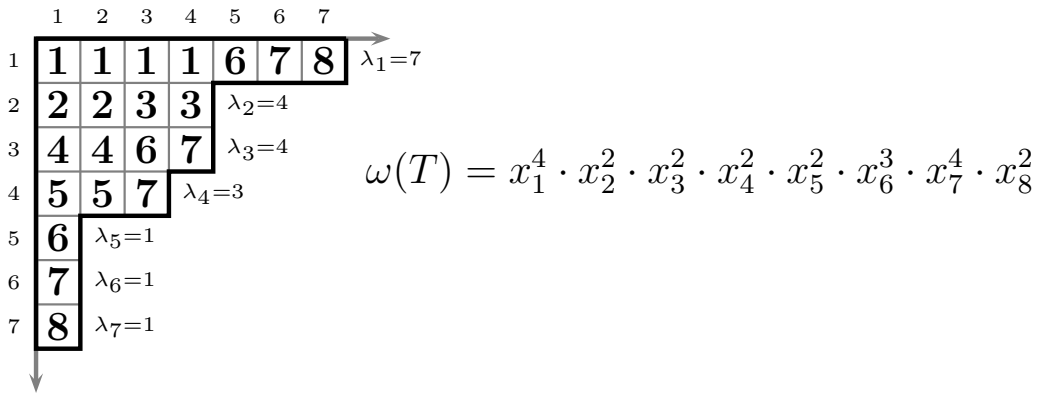

An $N$-semistandard Young tableau of shape $\lambda$ is a filling of the cells of the Ferrers diagram $F_{\lambda}$ with integers from the set $\{1,2, \ldots, N\}$, such that the numbers filled into the cells weakly increase in rows and strictly increase in columns.

Let $T$ be a semistandard Young tableau and define \# $(T, k)$ to be the number of entries $k$ in $T$. The weight $\omega(T)$ of $T$ is defined as follows:

$$
\omega(T)=\prod_{k=1}^{N} x_{k}^{\#(T, k)} .
$$

See Figure 3 for an illustration.

Then the Schur function $s_{\lambda}$ can equivalently be written as the following generating function (formal sum of weights)

$$
s_{\lambda}=\sum_{T} \omega(T)
$$

where the sum is over all $N$-semistandard Young tableaux $T$ of shape $\lambda$ (see [22, Definition 4.4.1]).

An $N$-semistandard skew Young tableau of shape $\lambda / \mu$ is a filling of the cells of $F_{\lambda / \mu}$ with integers from the set $\{1,2, \ldots, N\}$, such that the numbers filled into the cells weakly increase in rows and strictly increase in columns. See the left picture in Figure 5 (or Figure 3 again) for an illustration.

Then we define the skew Schur function $s_{\lambda / \mu}$ as the following generating function:

$$
s_{\lambda / \mu}:=\sum_{T} \omega(T)
$$

where the sum is over all $N$-semistandard skew Young tableaux $T$ of shape $\lambda / \mu$, where the weight $\omega(T)$ of $T$ is defined as in (4). The function $s_{\lambda / \mu}$ also is a symmetric function (see 
[22, proof of Proposition 4.4.2]), which is homogeneous of degree $|\lambda / \mu|$. Let $m:=\ell(\lambda / \mu)$, then by (1), we clearly have

$$
\begin{aligned}
& s_{\lambda / \mu}=s_{\lambda+(\bar{z}) / \mu+(\bar{z})} \text { for arbitrary } z \in \mathbb{Z}, \\
& s_{\lambda / \mu}=s_{\lambda+\left(z^{\lfloor m\rceil}\right) / \mu+\left(z^{\lfloor m\rceil}\right)} \text { for } z>0 \in \mathbb{Z} .
\end{aligned}
$$

In particular, $s_{\lambda}=s_{\lambda+(\bar{z}) /(\bar{z})}$ for arbitrary $z \in \mathbb{Z}$, and $s_{\lambda}=s_{\lambda+\left(z^{\lfloor m\urcorner}\right) /\left(z^{\lfloor m\urcorner}\right)}$ for $z>0 \in \mathbb{Z}$.

Skew Schur functions, too, have an expansion in terms of complete homogeneous symmetric functions (see $[19,(5.4)]$ ) which generalizes (3):

$$
s_{\lambda / \mu}=\left|h_{\lambda_{j}-\mu_{i}-j+i}\right|_{i, j=1}^{m} .
$$

\subsection{Connection between determinantal relations and skew Schur function identities}

We want to illustrate the connection between determinants and skew Schur functions, using Dodgson's condensation formula [4] (see also [20, Vol. III, page 17]) as an example.

Remark 1. Dodgson's condensation formula is also known as the Desnanot-Jacobi adjoint matrix theorem: According to Bressoud [3, remarks following Theorem 3.12], Lagrange [16] discovered this theorem for $n=3$ in 1773 (see also [20, vol. I, p. 37ff]), Desnanot proved it for $n \leqslant 6$ in 1819 (see [20, vol. I, p. 142]), and Jacobi [11] published the general theorem in 1841 (see also [20, vol. I, p. 264]).

Consider some $m \times n$-matrix

$$
a=\left(a_{i, j}\right)_{(i, j)=(1,1)}^{(m, n)}
$$

and define $[k]:=\{1,2, \ldots, k\}$ for $k \in \mathbb{N}$. Then we may write

$$
a=(a)_{[m],[n]}:=\left(a_{i, j}\right)_{(i, j) \in[m] \times[n]} .
$$

More generally, for subsets $R \subseteq[m]$ and $C \subseteq[n]$, we denote by $(a)_{R, C}$ the submatrix of $a$ which consists of the rows $R$ and the columns $C$; in the same order as in $a$. (All sets considered in this paper are ordered, and all subsets inherit the order from their supersets.)

If we want to describe the same submatrix by deleting rows and columns in $a$, then we write

$$
(a)_{R, C}=(a)_{\bar{X}, \bar{Y}},
$$

where $X=\bar{R}:=[m] \backslash R$ and $Y=\bar{C}:=[n] \backslash C$.

Proposition 2 (Dodgson's Condensation). Let $a=\left(a_{i, j}\right)_{i, j=1}^{m}$ be an arbitrary $m \times m$ matrix, $m \geqslant 2$. Then there holds the following identity:

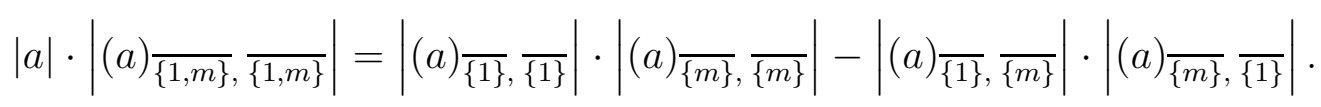

We adopt the convention that the empty determinant equals 1, i.e., $\left|(a)_{\emptyset, \emptyset}\right| \equiv 1$ : Then for $m=2$, (8) simply amounts to the formula for $2 \times 2$-determinants. 
We shall "translate" this determinantal identity to a Schur function identity. To this end, we introduce the operation "delete part $\lambda_{k}$ in semipartition $\lambda$ ", which we denote by $\lambda^{\bar{k}}$ :

$$
\lambda^{\bar{k}}:=\left(\lambda_{1}, \ldots, \lambda_{k-1}, \lambda_{k+1}-1, \lambda_{k+2}-1, \ldots\right) .
$$

Note that $\lambda_{\infty}^{\bar{k}}=\lambda_{\infty}-1$ and $\ell\left(\lambda^{\bar{k}}\right)=\max (\ell(\lambda), k)-1$.

For some subset $\left\{k_{1}<k_{2}<\cdots<k_{l}\right\} \subseteq\{1,2, \ldots, m\}$, we define inductively:

$$
\lambda^{\overline{k_{1}, \ldots, k_{l}}}:=\left(\lambda^{\overline{k_{2}, \ldots, k_{l}}}\right)^{\overline{k_{1}}}
$$

Now assume some skew shape $\lambda / \mu, m=\ell(\lambda / \mu)$. According to (7), the skew Schur function $s_{\lambda / \mu}$ equals the determinant of the matrix

$$
\mathbf{h}_{\lambda / \mu}:=\left(h_{\lambda_{j}-\mu_{i}-j+i}\right)_{(i, j)=1,1}^{(m, m)} .
$$

Observe that the $(i, j)$-entries in matrix $\left(\mathbf{h}_{\lambda / \mu}\right)_{\bar{\emptyset}, \overline{\{k\}}}$ are

- $h_{\lambda_{j}-\mu_{i}-j+i}$ for $j<k$,

- $h_{\left(\lambda_{j+1}-1\right)-\mu_{i}-j+i}$ for $k \leqslant j \leqslant m-1$,

i.e., deleting column $k$ in $\mathbf{h}_{\lambda / \mu}$ corresponds to deleting $\lambda_{k}$ in $\lambda$. Likewise, the $(i, j)$-entries in matrix $\left(\mathbf{h}_{\lambda / \mu}\right)_{\overline{\{l\}}, \bar{\emptyset}}$ are

- $h_{\lambda_{j}-\mu_{i}-j+i}$ for $i<l$,

- $h_{\lambda_{j}-\left(\mu_{i+1}-1\right)-j+i}$ for $l \leqslant i \leqslant m-1$,

i.e., deleting row $l$ in $\mathbf{h}_{\lambda / \mu}$ corresponds to deleting $\mu_{l}$ in $\mu$. These observations generalize to the following relation:

$$
\left(\mathbf{h}_{\lambda / \mu}\right)_{\overline{\left\{i_{1}, \ldots, i_{k}\right\}}}, \overline{\left\{j_{1}, \ldots, j_{l}\right\}}=\mathbf{h}_{\lambda^{\overline{i_{1}, \ldots, i_{k}} / \mu}} \overline{\mu^{j_{1}, \ldots, j_{l}}} .
$$

So from (8) we immediately deduce the following Schur function identity (11). To provide a combinatorial proof for (11) (in fact, a special case which was considered in [14]) was the starting point for the work in [6].

Corollary 1. Let $\lambda=\left(\lambda_{1}, \lambda_{2}, \ldots, \lambda_{m}\right)$ be a partition of length $\ell(\lambda)=m \geqslant 2$. Then we have the following Schur function identiy:

$$
\begin{aligned}
& s_{\lambda} \cdot s_{\left(\lambda_{2}, \lambda_{3}, \ldots, \lambda_{m-1}\right)}= \\
& s_{\left(\lambda_{2}, \lambda_{3}, \ldots, \lambda_{m}\right)} \cdot s_{\left(\lambda_{1}, \lambda_{2}, \ldots, \lambda_{m-1}\right)}-s_{\left(\lambda_{2}-1, \lambda_{3}-1, \ldots, \lambda_{m}-1\right)} \cdot s_{\left(\lambda_{1}+1, \lambda_{2}+1, \ldots, \lambda_{m-1}+1\right)}
\end{aligned}
$$


Proof. Consider the matrix $a=\mathbf{h}_{\left(\lambda+\left(2^{\lfloor m\rceil}\right)\right) /\left(2^{\lfloor m\rceil}\right)}$ (which is equal to $\mathbf{h}_{\lambda}$ ). Then (8) translates to

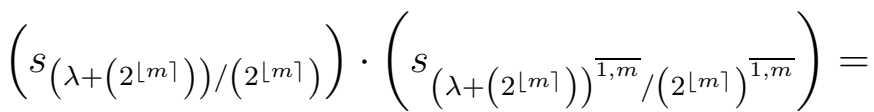

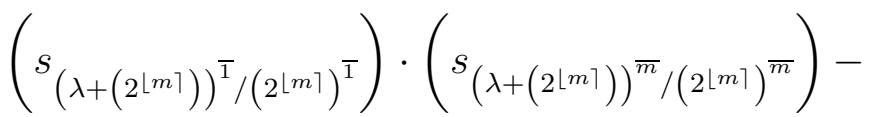

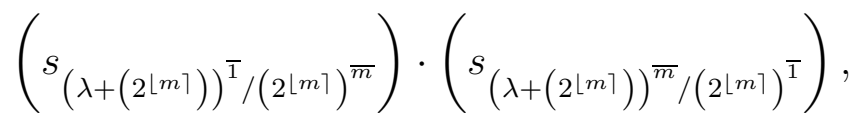

by (7) and (10). By (6) this simplifies to (11).

So it was easy to prove the Schur function identity (11) by recognizing it as a special case of the general determinantal identity (8). But this also works the other way round:

Observation 1 (Schur function identities imply equivalent determinantal identities). Assume that we have an identity $\mathcal{S}$ involving skew Schur functions.

Then by (7) and Proposition 1, $\mathcal{S}$ translates to a determinantal identity $\mathcal{D}$ which is equivalent to $\mathcal{S}$ as follows:

- Rewrite each skew Schur function in $\mathcal{S}$ as a determinant of complete homogeneous symmetric functions, according to (7),

- and then replace each entry $h_{r}$ by variable $y_{r}$, where $\left(y_{r}\right)_{r=0}^{\infty}$ is a set of independent variables, according to Proposition 1.

We call (skew) Schur function identities which are valid for arbitrary shapes $\lambda / \mu$ generic (skew) Schur function identities: Note that (11) is a generic identity in this sense. All identities we shall consider in the rest of this paper are generic.

In particular, we may apply (11) to $\lambda=\left(\lambda_{1}, \ldots, \lambda_{m}\right)$, where $\lambda_{j}=m \cdot(m-j+1)$. It is easy to see that for this choice of $\lambda$, all the entries in $\left(h_{\lambda_{j}-j+i}\right)_{i, j=1}^{m}$ are distinct, whence we may replace them by independent variables (by Proposition 1). This means that (11) implies the general determinantal identity (8): The identities are, in fact, equivalent.

It is clear, that this phenomenon will apply also to other determinantal identities: In this paper, we shall present generic Schur function identities which are equivalent to classical determinantal identities, and give combinatorial proofs for these Schur function identities.

\section{$3 \quad$ Determinants as nonintersecting lattice paths}

The Lindström-Gessel-Viennot-method $[8,7,13,18]$ is well-known: But since we want to present a "nonintersecting lattice path"-proof of the Cauchy-Binet formula (21) which involves a slight generalization, we repeat this beautiful idea here in some detail, using the Jacobi-Trudi-determinant as an illustrating example. 


\subsection{Lattice paths}

Consider the square lattice $\mathbb{Z}^{2}$, i.e., the directed graph with vertices $\mathbb{Z} \times \mathbb{Z}$ (we shall call them points), where the set of arcs consists of

- horizontal arcs $a_{(j, k)}^{h}$ from $(j, k)$ to $(j+1, k)$ for $j, k \in \mathbb{Z}$, and

- vertical arcs $a_{(j, k)}^{v}$ from $(j, k)$ to $(j, k+1)$ for $j, k \in \mathbb{Z}$.

Assign to these arcs the following weights:

$$
\begin{aligned}
& \left.\omega\left(a_{(j, k)}^{v}\right):=1 \text { (i.e., vertical arcs have weight } 1\right) \\
& \omega\left(a_{(j, k)}^{h}\right):=x_{k} \text { (i.e., horizontal arcs have weight } x_{\text {height of the arc }) .}
\end{aligned}
$$

A lattice path $p$ of length $k$ connecting starting point $v$ to ending point $w$ is a sequence of points $\left(v=v_{0}, v_{1}, \ldots, v_{k}=w\right)$, such that $\left(v_{i-1}, v_{i}\right)$ is a (horizontal or vertical) arc $a_{i}$ for $i=1,2, \ldots, k$. We say that all these arcs $a_{i}$ and points $v_{i}$ belong to the path $p$ and write $a_{i} \in p$ and $v_{i} \in p$. The weight of $p$ is defined as the product of the weights of the arcs belonging to $p$

$$
\omega(p)=\prod_{a \in p} \omega(a)
$$

Denote by $\mathfrak{P}(v \rightarrow w)$ the set of all lattice paths connecting starting point $v$ to ending point $w$. Observe that we may view the complete homogeneous symmetric function

$$
h_{m}\left(x_{j}, x_{j+1}, \ldots, x_{k}\right), k \geqslant j
$$

as the generating function of $\mathfrak{P}(v \rightarrow w)$, where $v=(t, j)$ and $w=(t+m, k)$ and $t \in \mathbb{Z}$ is an arbitrary shift parameter:

$$
\operatorname{gf}(\mathfrak{P}(v \rightarrow w))=\sum_{p \in \mathfrak{P}(v \rightarrow w)} \omega(p)
$$

I.e., the sum is over all paths $p$ connecting $v$ and $w$. (See Figure 4.)

Note that the ending point of some path $p$ never lies below (or to the left of) its starting point: From now on, we shall call

- the starting point $p$ its lower point,

- and the ending point $p$ its upper point.

This change of nomenclature seems pointless here, but later we shall also consider trails in the undirected graph $\mathbb{Z}^{2}$ which connect starting and ending points of paths: A trail $p^{\prime}$ of length $k$ connecting point $v$ to point $w$ is a sequence of points $\left(v=v_{0}, v_{1}, \ldots, v_{k}=w\right)$, such that $\left(v_{i-1}, v_{i}\right)$ or $\left(v_{i}, v_{i-1}\right)$ is a (horizontal or vertical) arc $\alpha_{i}$ for $i=1,2, \ldots, k$ (i.e., $p^{\prime}$ may use arcs "in the wrong direction"). For such trail, it is not clear whether $v$ or $w$ is the starting or ending point. 
Figure 4: Complete homogeneous symmetric functions may be viewed as generating functions of lattice paths with fixed starting (lower) and ending (upper) point. The picture below illustrates this for $h_{8}\left(x_{5}, x_{6}, \ldots, x_{13}\right)$, which appears as the sum of the weights of all lattice paths connecting starting point $(-4,5)$ to ending point $(4,13)$, showing the lattice paths associated to the two monomials $\left(x_{5} \cdot x_{6}^{4} \cdot x_{7} \cdot x_{9}^{2}\right)$ and $\left(x_{10} \cdot x_{11}^{2} \cdot x_{12}^{4} \cdot x_{13}\right)$ in $h_{8}\left(x_{5}, x_{6}, \ldots, x_{13}\right)$. Note that we may shift the picture horizontally by an arbitrary vector $(t, 0), t \in \mathbb{Z}$, without changing the generating function of the lattice paths.

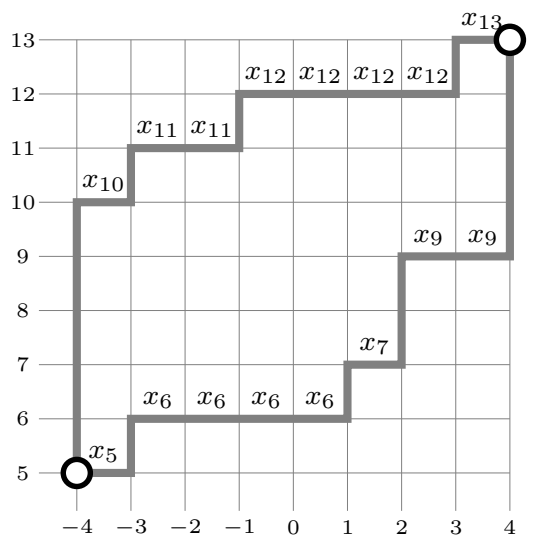

\subsection{Young tableaux and nonintersecting lattice paths}

The following weight-preserving bijection gives an equivalent description of a semistandard Young tableau $T$ of shape $\lambda / \mu$ as an $m$-tuple $P=\left(p_{1}, \ldots, p_{m}\right)$ of nonintersecting lattice paths, where $m:=\ell(\lambda / \mu)$. The $i$-th path $p_{i}$ starts at lower point $\left(\mu_{i}-i, 1\right)$ and ends at upper point $\left(\lambda_{i}-i, N\right)$ : We call these points the lower/upper points associated to the skew shape $\lambda / \mu$ (and we count these points always from the right). The $k$-th horizontal step in $p_{i}$ goes from $\left(\mu_{i}-i+k-1, x\right)$ to $\left(\mu_{i}-i+k, x\right)$, where $x$ is the $k$-th entry in row $i$ of $T$ (i.e., the heights of the horizontal steps in path $p_{i}$ are read off the $i$-th row of $T$ ).

Note that the conditions on the entries of $T$ imply that no two paths $p_{i}$ and $p_{j}$ thus defined have a lattice point in common if $i \neq j$ : Such an $m$-tuple of paths is called nonintersecting, see the right picture of Figure 5 for an illustration. An $m$-tuple of paths which is not nonintersecting is called intersecting.

In fact, this translation of tableaux to nonintersecting lattice paths is a bijection between the set of all $N$-semistandard Young tableaux of shape $\lambda / \mu$ and the set of all $\ell(\lambda / \mu)$-tuples of nonintersecting lattice paths with lower (starting) and upper (ending) points as defined above.

This bijection clearly is weight preserving if we define the weight of an $m$-tuple $P=$ $\left(p_{1}, \ldots, p_{m}\right)$ of lattice paths in the obvious way, i.e., as

$$
\omega(P):=\prod_{k=1}^{m} \omega\left(p_{k}\right)=\prod_{k=1}^{N} x_{k}^{\#(P, k)}
$$


Figure 5: The left picture presents a semistandard Young tableau $T$ of shape $\lambda / \mu$, where $\lambda=(9,6,6,5,3,3,3)$ and $\mu=(5,4,4,3,2,2,2)$. Assuming that the entries of $T$ are chosen from $\{1,2, \ldots, 8\}$ (i.e.: $T$ is an 8 -semistandard Young tableau), the right picture shows the corresponding family of $7=\ell(\lambda / \mu)$ nonintersecting lattice paths: Note that the height of the $k$-th horizontal step in the $i$-th path (the paths are counted from right to left) is equal to the $k$-th entry in row $i$ of $T$.
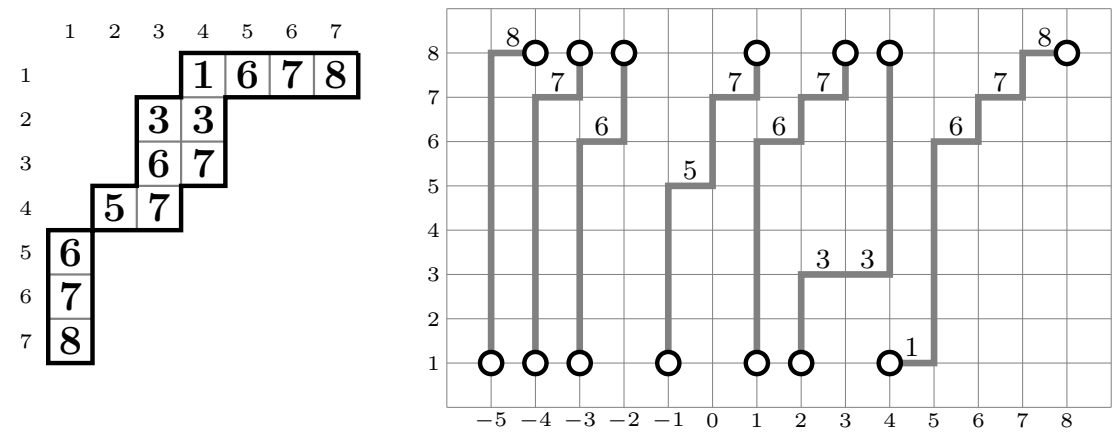

where $\#(P, k)$ is the number of horizontal steps at height $k$ in $P$. So in definition (5) we could equivalently replace symbol " $T$ " by symbol " $P$ ", and sum over $m$-tuples $P$ of nonintersecting lattice paths with prescribed lower and upper points instead of tableaux with prescribed shape:

$$
s_{\lambda / \mu}:=\sum_{P} \omega(P) .
$$

Note that the horizontal coordinates of lower and upper points determine uniquely the shape $\lambda / \mu$ of the tableau, and the vertical coordinates (we shall call the vertical coordinate of points the level in the following) of the ending points determines uniquely the set of entries $\{1,2, \ldots, N\}$ of the tableau: More generally, lower points at level $\alpha$ and upper points at level $\omega \geqslant \alpha$ would give rise to entries $\{\alpha, \alpha+1, \ldots, \omega\}$.

Observe that the operation of deleting part $i$ in $\mu=\left(\mu_{1}, \ldots, \mu_{m}\right)$ and part $j$ in $\lambda=$ $\left(\lambda_{1}, \ldots, \lambda_{m}\right)$ (defined in (9)) translates to the removal of the $i$-th lower point and the $j$-th upper point associated to $\lambda / \mu$, and by (10), this removal of lower/upper points translates to deleting row $i$ and column $j$ in $\mathbf{h}_{\lambda / \mu}$. Clearly, this generalizes to the following observation:

Observation 2. Minors of $\mathbf{h}_{\lambda / \mu}$ consisting of rows $\left\{i_{1}, \ldots, i_{k}\right\}$ and columns $\left\{j_{1}, \ldots, j_{l}\right\}$ are in one-to-one correspondence to the selection of

- lower points with indices in $\left\{i_{1}, \ldots, i_{k}\right\}$

- and upper points with indices in $\left\{j_{1}, \ldots, j_{l}\right\}$

from the points associated to the shape $\lambda / \mu$.

Together, Observations 1 and 2 explain the proper meaning of the statement given in Section 1. 


\subsection{The Lindström-Gessel-Viennot-proof of the Jacobi-Trudi identity}

Let $\lambda / \mu$ be some skew shape, let $m=\ell(\lambda / \mu)$, and consider the lower/upper points of the corresponding lattice paths. Denote the $i$-th lower point $\left(\mu_{i}-i, 1\right)$ by $s_{i}$, and the $j$-th upper point $\left(\lambda_{j}-j, N\right)$ by $t_{j}$. Observe that the entry $(i, j)$ in the matrix $\mathbf{h}_{\lambda / \mu}$ is the generating function

$$
\operatorname{gf}\left(\mathfrak{P}\left(s_{i} \rightarrow t_{j}\right)\right):=\sum_{p} \omega(p)=h_{\lambda_{j}-\mu_{i}-j+i},
$$

where the sum is over all lattice paths $p$ that run from $s_{i}$ to $t_{j}$, and where the weight $\omega(p)$ is defined as in (12). By expanding the determinant in (7), we thus obtain

$$
\left|h_{\lambda_{j}-\mu_{i}-j+i}\right|_{i, j=1}^{m}=\sum_{\pi \in \mathfrak{S}_{m}} \operatorname{sgn}(\pi) \cdot \prod_{j=1}^{m}\left(\mathbf{g} \mathbf{f}\left(\mathfrak{P}\left(s_{\pi_{j}} \rightarrow t_{j}\right)\right)\right) .
$$

Consider the following set

$$
\mathfrak{D}_{\lambda / \mu}:=\bigcup_{\pi \in \mathfrak{S}_{m}} \mathfrak{P}\left(s_{\pi_{1}} \rightarrow t_{1}\right) \times \mathfrak{P}\left(s_{\pi_{2}} \rightarrow t_{2}\right) \times \cdots \times \mathfrak{P}\left(s_{\pi_{m}} \rightarrow t_{m}\right)
$$

of $m$-tuples $P$ of lattice paths connecting the permuted lower points $\left(s_{\pi_{1}}, s_{\pi_{2}}, \ldots, s_{\pi_{m}}\right)$ with the upper points $\left(t_{1}, t_{2}, \ldots, t_{m}\right)$, where in addition to the weight $\omega(P)$, the elements of $\mathfrak{D}_{\lambda / \mu}$ also carry a sign which equals the sign of the corresponding permutation $\pi$ :

$$
\operatorname{sgn}(P)=\operatorname{sgn}(\pi) .
$$

Then (15) can be rewritten equivalently as the generating function of $\mathfrak{D}_{\lambda / \mu}$ :

$$
\left|h_{\lambda_{j}-\mu_{i}-j+i}\right|_{i, j=1}^{m}=\sum_{P \in \mathfrak{D}_{\lambda / \mu}} \operatorname{sgn}(P) \cdot \omega(P) .
$$

Denote by $\mathfrak{N}_{\lambda / \mu}$ the subset of nonintersecting $m$-tuples of lattice paths in $\mathfrak{D}_{\lambda / \mu}$. In order to prove (7), we only need to show that we do in fact have

$$
\left|h_{\lambda_{j}-\mu_{i}-j+i}\right|_{i, j=1}^{m}=\sum_{P \in \mathfrak{N}_{\lambda / \mu}} \operatorname{sgn}(P) \cdot \omega(P),
$$

since $P \in \mathfrak{N}_{\lambda / \mu}$ implies $\pi=$ id (i.e., all these $m$-tuples $P$ objects have sign +1 ) and $s_{\lambda / \mu}=\sum_{P \in \mathfrak{N}_{\lambda / \mu}} \operatorname{sgn}(P) \cdot \omega(P)$ by definition (14). This certainly would be achieved by showing that all the signed weights of $m$-tuples $P$ in $\mathfrak{I}_{\lambda / \mu}:=\mathfrak{D}_{\lambda / \mu} \backslash \mathfrak{N}_{\lambda / \mu}$ cancel in (17). To this end, we shall present an involution (a self-inverse bijective mapping) on intersecting $m$-tuples of lattice paths

$$
\mathbf{i}:\left(\mathfrak{I}_{\lambda / \mu}\right) \rightarrow\left(\mathfrak{I}_{\lambda / \mu}\right)
$$

which is 
- weight-preserving, i.e., $\omega(\mathbf{i}(P))=\omega(P)$

- and sign-reversing, i.e., $\operatorname{sgn}(\mathbf{i}(P))=-\operatorname{sgn}(P)$.

The construction of $\mathbf{i}$ is simple: Let $P$ be an intersecting $m$-tuple in $\mathfrak{I}_{\lambda / \mu}$. Consider the smallest point of intersection $q$ in $P$, in lexicographic order:

$$
(a, b) \leqslant(c, d): \Leftrightarrow a \leqslant b \vee(a=b \wedge b \leqslant c) .
$$

Observe that (by the minimality of $q$ ) there are precisely two paths $p_{k}$ and $p_{l}$ meeting in $q: \mathbf{i}(P)$ is obtained from $P$ by interchanging the initial segments (from lower points up to $q$ ) of $p_{k}$ and $p_{l}$, see Figure 6 for an illustration.

Figure 6: Illustration of the Lindström-Gessel-Viennot-involution i.

Both pictures show quadruples of lattice paths belonging to $\mathfrak{I}_{\lambda / \mu}$, where $\lambda=(8,6,4,3)$ and $\mu=(0)$. There are 4 points of intersections at positions

$$
(1,2)<(1,5)<(4,5)<(4,6)
$$

written in lexicographic order. (Note that the lattice paths are drawn with rounded corners and small offsets here, just to make obvious the course of the paths, which is not clear for intersecting paths). The smallest such point in lexicographic order is $q:=(1,2)$ (indicated by a circle). The right picture is obtained from the left picture by interchanging the initial segments (from lower points up to $q$ ) of the two paths intersecting in $q$, and vice versa. To the left picture, the identity permutation (i.e., sign +1 ) is associated, while to the right picture, the transposition $(3,4)$ (i.e., sign -1 ) is associated. This illustrates that in the determinantal expansion of $s_{\lambda}=\left|h_{\lambda_{j}-j+i}\right|$, the following terms cancel:

$$
\left(x_{7}^{3}\right)\left(x_{5}^{4}\right)\left(x_{2}^{3} x_{5}^{3}\right)\left(x_{1}^{2} x_{2} x_{3}^{2} x_{6}^{3}\right)-\left(x_{7}^{3}\right)\left(x_{5}^{4}\right)\left(x_{2}^{4} x_{3}^{2} x_{6}^{3}\right)\left(x_{1}^{2} x_{5}^{3}\right)=0 .
$$
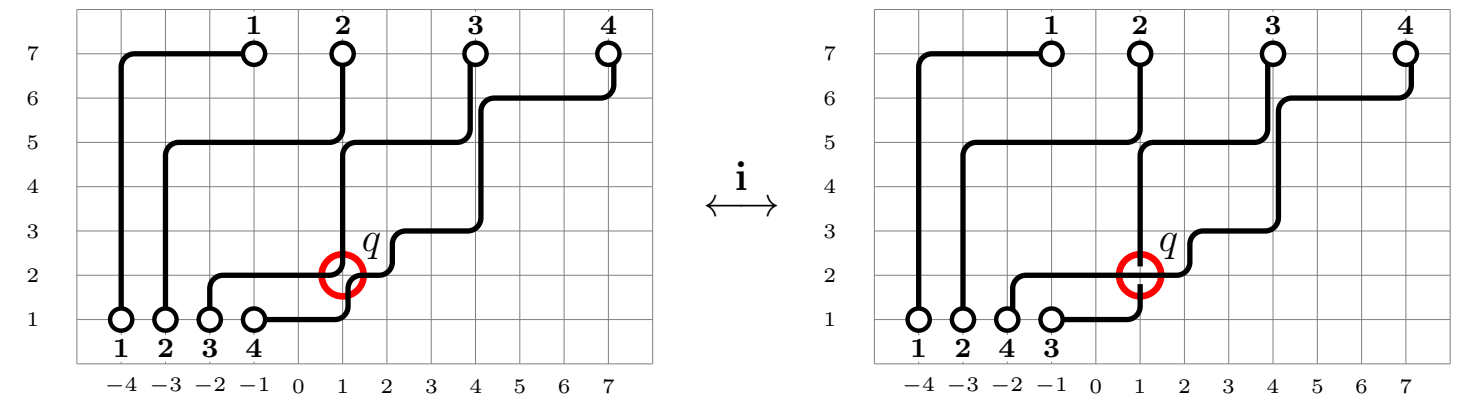

It is immediately clear that $\mathbf{i}$ is an involution which is weight-preserving and signreversing (since it modifies the original permutation associated to $P$ by the transposition corresponding to the swapping of the lower points of $p_{k}$ and $p_{l}$ ).

Clearly, i describes the pairwise cancellation

$$
\operatorname{sgn}(P) \cdot \omega(P)+\mathbf{i}(\operatorname{sgn}(P) \cdot \omega(P))=0
$$


of all objects from $\mathfrak{I}_{\lambda / \mu}$ in the sum (17). Stated otherwise: Only the nonintersecting objects in $\mathfrak{N}_{\lambda / \mu} \subseteq \mathfrak{D}_{\lambda / \mu}$ "survive", which proves (18).

\subsection{Viewing determinants as nonintersecting lattice paths}

Our considerations so far showed that the generic determinant $\left|a_{i, j}\right|_{i, j=1}^{m}$ ("generic" means that the entries $a_{i, j}$ are independent variables) may be viewed as a skew Schur function of appropriate shape $\lambda / \mu$ with $\ell(\lambda / \mu)=m$ ("appropriate" means that all entries in $\mathbf{h}_{\lambda / \mu}$ are distinct). Together with the the Lindström-Gessel-Viennot-view, the determinant $\left|a_{i, j}\right|_{i, j=1}^{m}$ appears as

- the generating function of m-tuples of nonintersecting lattice paths,

- where the rows (from top to bottom) correspond to the starting points of the lattice paths (from right to left),

- and where the columns (from left to right) correspond to the starting points of the lattice paths (from right to left).

We shall demonstrate the power of this point of view by giving a simple proof of the Cauchy-Binet formula (21), which becomes even more transparent if we prove the multiplicativity of the determinant function as a preparatory step. (A similar proof in an easily comprehensible exposition is given in [1].)

\subsubsection{Multiplicativity of the determinant function: A proof "by example".}

Given some arc $\alpha$ in the lattice $\mathbb{Z}^{2}$, we say that a path $p$ starts in $\alpha$ (has $\alpha$ as its lower arc), if $p$ starts in the upper (if $\alpha$ is vertical) or right (if $\alpha$ is horizontal) point of $\alpha$. Likewise, we say that $p$ ends in $\alpha$ (has $\alpha$ as its upper arc), if $p$ ends in the lower (if $\alpha$ is vertical) or left (if $\alpha$ is horizontal) point of $\alpha$.

We want to illustrate the multiplicativity of the determinant function

$$
|a \cdot b|=|a| \cdot|b|
$$

by considering the special case

$$
a:=\left(h_{\lambda_{j}-j+i}\left(x_{1}, x_{2}, \ldots x_{7}\right)\right)_{i, j=1}^{4} \text { and } b:=\left(h_{\sigma_{j}-\lambda_{i}-j+i}\left(x_{8}, x_{9} \ldots x_{13}\right)\right)_{i, j=1}^{4},
$$

where $\lambda=(8,6,4,3)$ and $\sigma=(18,16,13,11)$.

By the weight-preserving bijection between Young tableaux and nonintersecting lattice paths, we may view $|a|$ as the generating function of quadruples of nonintersecting lattice paths connecting lower points $\mathbf{r}=\left(r_{1}, r_{2}, r_{3}, r_{4}\right)$ with upper $\operatorname{arcs} \mathbf{s}=\left(s_{1}, s_{2}, s_{3}, s_{4}\right)$, where

$$
\begin{aligned}
& \mathbf{r}=((-1,1),(-2,1),(-3,1),(-4,1)), \\
& \mathbf{s}=(((7,7),(7,8)),((4,7),(4,8)),((1,7),(1,8)),((-1,7),(-1,8))) .
\end{aligned}
$$


Figure 7: Illustration: Multiplicativity of the determinant. The picture shows the lattice paths corresponding to the monomial

$$
\left(\left(x_{7}^{3}\right) \cdot\left(x_{9}^{2} x_{11} x_{13}^{5}\right)\right) \cdot\left(\left(x_{5}^{4}\right) \cdot\left(x_{8}^{2} x_{11}^{5} x_{12}^{2}\right)\right) \cdot\left(\left(x_{2}^{3} x_{4}^{3}\right) \cdot\left(x_{9}^{5} x_{11}^{5}\right)\right) \cdot\left(\left(x_{1}^{3} x_{3}^{4} x_{5}\right) \cdot\left(x_{8}^{4} x_{10}^{6}\right)\right),
$$

which appears in the expansion of the product of skew Schur functions

$$
s_{\lambda}\left(x_{1}, x_{2}, \ldots x_{7}\right) \cdot s_{\sigma / \lambda}\left(x_{8}, x_{9}, \ldots x_{13}\right),
$$

where $\lambda=(8,6,4,3)$ and $\sigma=(18,16,13,11)$.

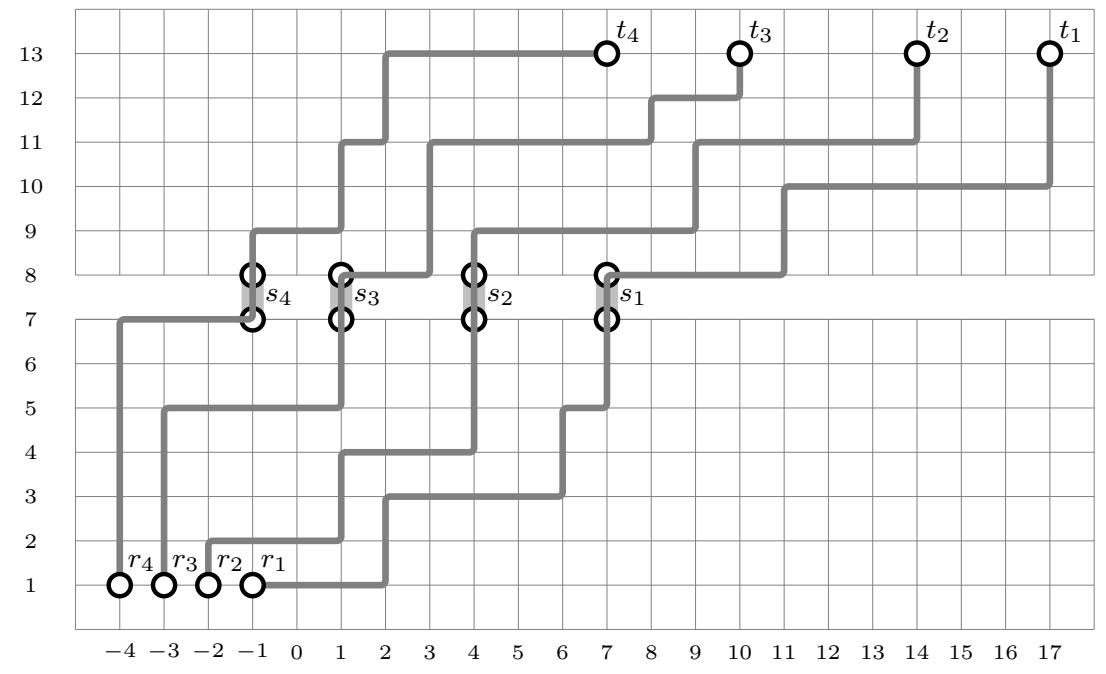

Likewise, we may view $|b|$ as the generating function of quadruples of nonintersecting lattice paths connecting lower arcs $\mathbf{s}$ (as above: Note that the set of variables involved in $b$ is $\left.\left\{x_{8}, \ldots, x_{13}\right\} !\right)$ with ending points $\mathbf{t}=\left(t_{1}, t_{2}, t_{3}, t_{4}\right)$, where

$$
\mathbf{t}=((17,13),(14,13),(10,13),(7,13)) \text {. }
$$

See Figure 3.4.1 for an illustration.

So the $(i, j)$-entry in $a \cdot b$ is

$$
\sum_{k=1}^{4} \mathbf{g f}\left(\mathfrak{P}\left(r_{i} \rightarrow s_{k}\right)\right) \cdot \mathbf{g f}\left(\mathfrak{P}\left(s_{k} \rightarrow t_{j}\right)\right)
$$

which may be viewed as the generating function $\mathbf{g f}\left(\mathfrak{P}^{\prime}\left(r_{i} \rightarrow t_{j}\right)\right)$ of the following set of constrained paths (see Figure 3.4.1 for an illustration):

$$
\mathfrak{P}^{\prime}\left(r_{i} \rightarrow t_{j}\right):=\bigcup_{k=1}^{4}\left\{p: p \text { is a lattice path connecting } r_{i} \text { to } t_{j} \text { passing through } s_{k}\right\}
$$


Figure 8: Illustration: The Lindström-Gessel-Viennot-involution for the "constrained" lattice paths $\mathbf{g f}\left(\mathfrak{P}^{\prime}\left(r_{i} \rightarrow t_{j}\right)\right)$ : The fact that every constrained path must pass through one of the arcs $s_{1}, s_{2}, s_{3}$ or $s_{4}$ is indicated by omitting all other arcs connecting level 7 and level 8. The picture shows a path connecting $r_{1}$ to $t_{1}$ which passes through the arc $s_{2}$, and a path connecting $r_{4}$ to $t_{3}$, which passes through the arc $s_{3}$. The smallest point of intersection in lexicographic order is $q:=(-4,9)$ (indicated by a circle; as in Figure 6 , the lattice paths are drawn with rounded corners here, just to make obvious the run of the paths.) Clearly, the Lindström-Gessel-Viennot-involution gives a path connecting $r_{1}$ to $t_{3}$ which passes through $s_{2}$, and a path connecting $r_{4}$ to $t_{1}$ which passes through $s_{3}$.

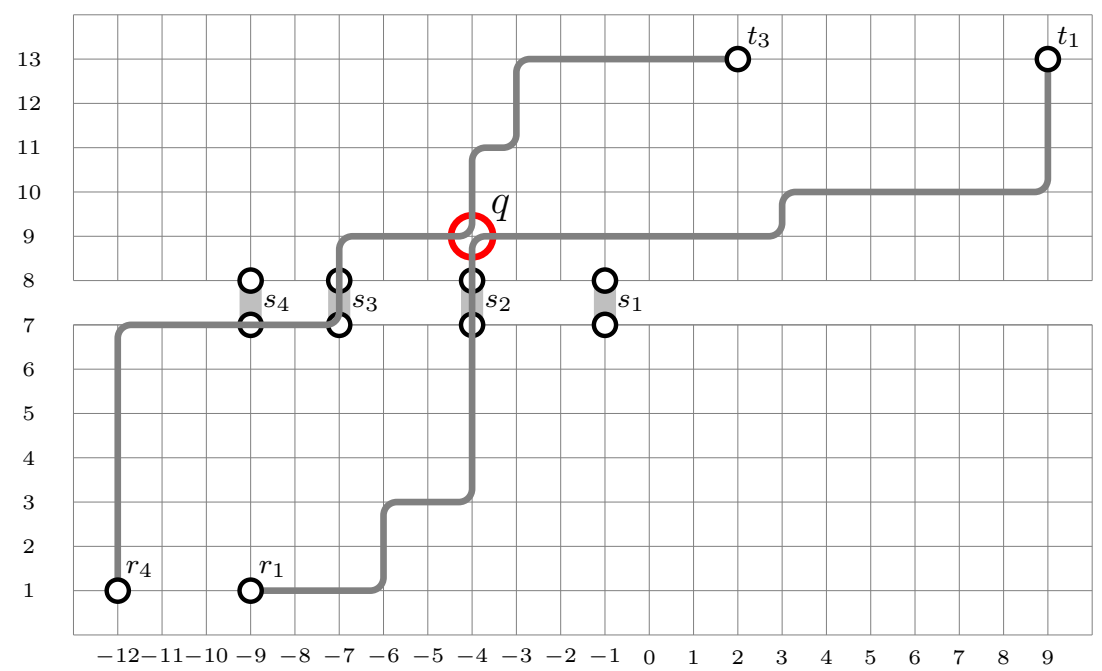

So as in the above proof of the Jacobi-Trudi identity, $|a \cdot b|$ can be rewritten equivalently as the generating function of the set $\mathfrak{D}^{\prime}$ of quadruples of constrained paths,

$$
\mathfrak{D}^{\prime}:=\bigcup_{\pi \in \mathfrak{S}_{4}} \mathfrak{P}^{\prime}\left(s_{\pi_{1}} \rightarrow t_{1}\right) \times \mathfrak{P}^{\prime}\left(s_{\pi_{2}} \rightarrow t_{2}\right) \times \mathfrak{P}^{\prime}\left(s_{\pi_{3}} \rightarrow t_{3}\right) \times \mathfrak{P}^{\prime}\left(s_{\pi_{4}} \rightarrow t_{4}\right),
$$

i.e., as

$$
|a \cdot b|=\mathbf{g} \mathbf{f}\left(\mathfrak{D}^{\prime}\right)=\sum_{P \in \mathfrak{D}^{\prime}} \operatorname{sgn}(P) \cdot \omega(P) .
$$

Denote by $\mathfrak{N}^{\prime}$ the subset of nonintersecting $m$-tuples of constrained lattice paths in $\mathfrak{D}^{\prime}$. Clearly, the Lindström-Gessel-Viennot-operation $\mathbf{i}$ can be applied to intersecting $m$ tuples of constrained lattice paths (see Figure 8 for an illustration) and establishes a weight-preserving and sign-reversing involution, i.e.,

$$
|a \cdot b|=\mathbf{g f}\left(\mathfrak{N}^{\prime}\right)
$$

On the other hand, $\mathfrak{N}^{\prime}$ appears (just look at Figure 3.4.1!) as the Cartesian product of

- the set of nonintersecting quadruples of lattice paths connecting $\mathbf{r}$ and $\mathbf{s}$ 
- and the set of nonintersecting quadruples of lattice paths connecting $\mathbf{s}$ and $\mathbf{t}$,

i.e.,

$$
\mathbf{g f}\left(\mathfrak{N}^{\prime}\right)=|a| \cdot|b|
$$

We presented our argument for a specific choice of partitions $\lambda \unlhd \sigma$ to make it more tangible, but it is clear that it holds for arbitrary choices of (semi)partitions. So fix $m>0$ and consider the following partitions $\lambda$ and $\sigma, \ell(\lambda)=\ell(\sigma)=m$ :

$$
\begin{aligned}
& \lambda=\left((m \cdot(m-1),(m-1) \cdot(m-1), \ldots,(m-1))\left(\text { i.e., } \lambda_{j}=(m-j+1) \cdot(m-1)\right),\right. \\
& \sigma=\left((m \cdot m)^{\lfloor m\rceil}\right)=\left(m^{2}, m^{2}, \ldots, m^{2}\right) \quad\left(\text { i.e., } \sigma_{i} \equiv m^{2}\right) .
\end{aligned}
$$

Then the entries in $a:=\mathbf{h}_{\lambda}\left(x_{1}, \ldots, x_{N}\right)$ and in $b:=\mathbf{h}_{\sigma / \lambda}\left(x_{N+1}, \ldots, x_{2 N}\right)$ are all distinct. Hence (by Observation 1) the identity

$$
|a \cdot b|=|a| \cdot|b|
$$

is equivalent with the multiplicativity of the determinant function in general.

\subsubsection{The Cauchy-Binet formula: Another proof "by example".}

Now it is "almost immediate" to see the Cauchy-Binet formula as an obvious generalization of the multiplicativity of the determinant.

Theorem 1 (Cauchy-Binet formula). Let a be an $m \times n$-matrix and $b$ be an $n \times m$-matrix. Then we have

$$
|a \cdot b|=\sum_{S \subseteq[n],|S|=m}\left|(a)_{[m], S}\right| \cdot\left|(b)_{S,[m]}\right| .
$$

Note that this formula holds trivially if $m>n$ (since the determinant $|a \cdot b|$ is zero in this case, as is the empty sum in (21)), and amounts to the multiplicativity of the determinant if $m=n$.

We illustrate this formula by a special case and consider the matrices

$$
a:=\left(h_{\lambda_{j}-j+i}\left(x_{1}, \ldots x_{7}\right)\right)_{(i, j) \in([3] \times[4])} \text { and } b:=\left(h_{\sigma_{j}-\lambda_{i}-j+i}\left(x_{8}, \ldots x_{13}\right)\right)_{(i, j) \in([4] \times[3])},
$$

where $\lambda=(9,7,5,4)$ and $\sigma=(19,17,14)$.

Again, we want to employ the weight-preserving bijection between Young tableaux and nonintersecting lattice paths. To this end, we consider the vectors of points $\mathbf{r}=\left(r_{1}, r_{2}, r_{3}\right)$ and $\mathbf{t}=\left(t_{1}, t_{2}, t_{3}\right)$, and the vector of arcs, $\mathbf{s}=\left(s_{1}, s_{2}, s_{3}, s_{4}\right)$, where

$$
\begin{aligned}
& \mathbf{r}=((-1,1),(-2,1),(-3,1)), \\
& \mathbf{s}=(((8,7),(8,8)),((5,7),(5,8)),((2,7),(2,8)),((0,7),(0,8))), \\
& \mathbf{t}=((18,13),(15,13),(11,13)),
\end{aligned}
$$

(See Figure 9 for an illustration.) 
Figure 9: Illustration: The Cauchy-Binet formula. Triples of nonintersecting lattice paths connecting points $\mathbf{r}=\left(r_{1}, r_{2}, r_{3}\right)$ and $\mathbf{t}=\left(t_{1}, t_{2}, t_{3}\right)$, where each single path must pass through an arc in $\mathbf{s}=\left(s_{1}, s_{2}, s_{3}, s_{4}\right)$, can be "cut in two halves" along $\mathbf{s}$. Note that the arcs used by the paths constitute a 3-element subvector $\mathbf{s}^{\prime}$, and the halves appear as tripels of nonintersecting lattice paths $\mathfrak{N}\left(\mathbf{r} \rightarrow \mathbf{s}^{\prime}\right)$ and $\mathfrak{N}\left(\mathbf{s}^{\prime} \rightarrow \mathbf{t}\right)$, respectively. In the picture, we have $\mathbf{s}^{\prime}=\left(s_{1}, s_{2}, s_{4}\right)$.

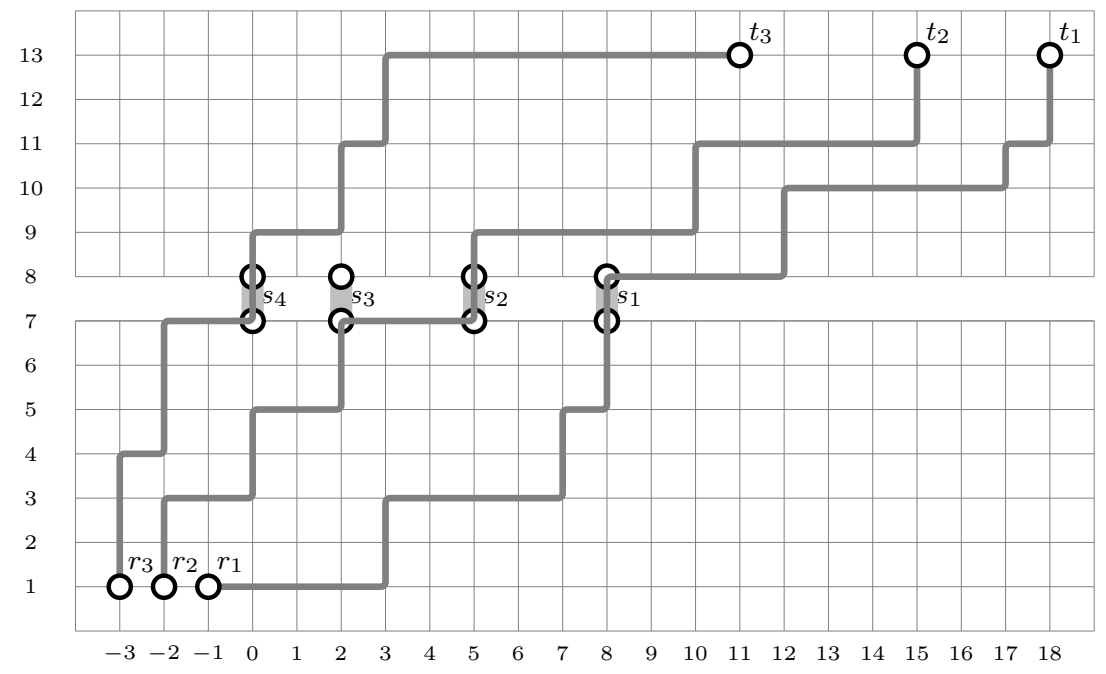

As before, observe that the $(i, j)$-entry in $a \cdot b$ may be viewed as the generating function $\operatorname{gf}\left(\mathfrak{P}\left(r_{i} \rightarrow t_{j}\right)\right)^{\prime}$ of constrained paths which must pass through one arc in $\mathbf{s}$. By the Lindström-Gessel-Viennot-involution, the determinant $|a \cdot b|$ appears as the generating function of triples of nonintersecting labeled paths $\mathfrak{N}^{\prime}(\mathbf{r} \rightarrow \mathbf{t}$ ), which (as in Section 3.4.1 — just look at Figure 9!) appears as

$$
\mathfrak{N}^{\prime}(\mathbf{r} \rightarrow \mathbf{t})=\bigcup_{\mathbf{s}^{\prime}}\left(\mathfrak{N}\left(\mathbf{r} \rightarrow \mathbf{s}^{\prime}\right) \times \mathfrak{N}\left(\mathbf{s}^{\prime} \rightarrow \mathbf{t}\right),\right)
$$

where the union runs over all 3-element subvectors $\mathbf{s}^{\prime}$ of $\mathbf{s}$. By the same reasoning as in Section 3.4.1, this shows (21).

\section{Products of determinants/Overlays of lattice paths}

In the following, all skew Schur functions are considered as functions of the same set of variables $\left(x_{1}, \ldots, x_{N}\right)$. (Equivalently, all tableaux have entries from the set $\{1, \ldots, N\}$, and all families of nonintersecting lattice paths have lower points on level 1 and upper points on level $N)$.

By (14) we may view the product of two skew Schur functions as the generating 
Figure 10: Illustration: A green 7-tuple of nonintersecting lattice paths, corresponding to shape $\lambda / \mu$, and a red 7 -tuple of nonintersecting lattice paths, corresponding to shape $\sigma / \tau$, constitute an overlay of nonintersecting lattice paths. Here, $\sigma=(7,4,4,3,1,1,1)$, $\tau=(3,2,2,1), \lambda=\sigma+\left(1^{\lfloor 7\rceil}\right)$ and $\mu=\tau+\left(1^{\lfloor 7\rceil}\right)$. (Note that $s_{\lambda / \mu}=s_{\sigma / \tau}$.) The red and green paths are drawn with a slight offset for graphical reasons, the colour red is indicated by dashed lines, the colour green is indicated by dotted lines. The picture also shows the Young tableaux corresponding to the red and green 7 -tuples of nonintersecting lattice paths.

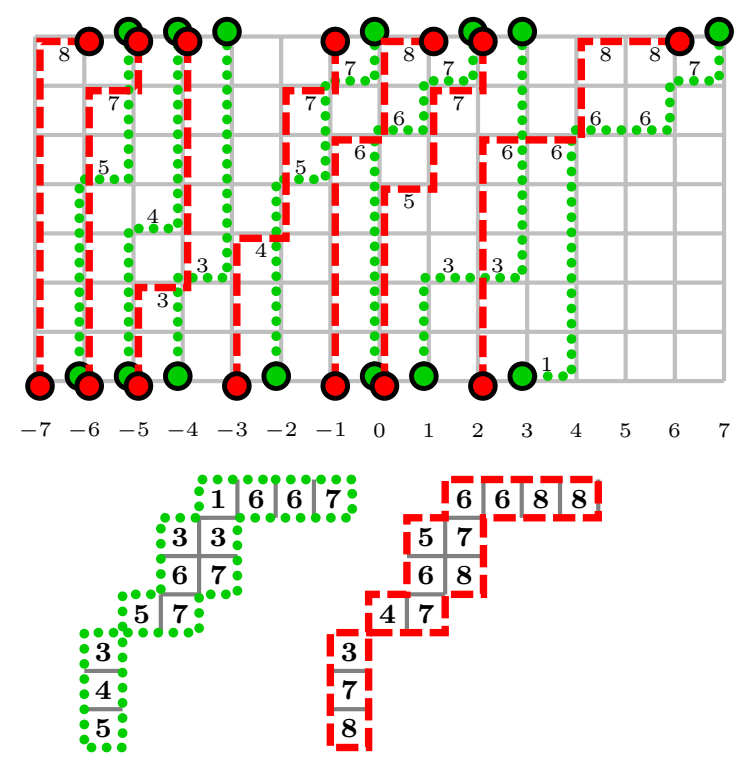

function of pairs of $m$-tuples of nonintersecting lattice paths

$$
s_{\lambda / \mu} \cdot s_{\sigma / \tau}=\sum_{\left(P_{1}, P_{2}\right)} \omega\left(P_{1}\right) \cdot \omega\left(P_{2}\right)
$$

Here, the sum runs over all pairs $\left(P_{1}, P_{2}\right)$, where $P_{1}$ is a $\ell(\lambda / \mu)$-tuple of nonintersecting lattice paths corresponding to the shape $\lambda / \mu$ and $P_{2}$ is a $\ell(\sigma / \tau)$-tuple of nonintersecting lattice paths corresponding to the shape $\sigma / \tau$. Imagine that the lattice paths of $P_{1}$ and $P_{2}$ are coloured red and green, respectively: This will give an overlay of lattice paths, see Figure 10 for an illustration.

Such overlays give rise to a bijective construction, which (to the best of our knowledge) was first used by Goulden [9]. The same construction was used in [6] to describe a class of Schur function identities, special cases of which amount to Dodgson's condensation formula and the Plücker relations.

We shall present this construction by way of an example: Consider skew shapes $\lambda / \mu$ and $\sigma / \tau$, where $\sigma=(7,4,4,3,1,1,1), \tau=(3,2,2,1), \lambda=\sigma+\left(1^{\lfloor 7\rceil}\right)$ and $\mu=\tau+\left(1^{\lfloor 7\rceil}\right)$, 
see Figure 10 for an illustration.

Now consider the arcs, lower points and upper points of some pair $\left(P_{1}, P_{2}\right)$ of 7 -tuples of lattice paths, where $P_{1}$ corresponds to shape $\lambda / \mu$ and $P_{2}$ corresponds to shape $\sigma / \tau$. Note that there may be arcs/points coloured both green and red. Such arcs/points will never be affected by the following constructions: We call them uncoloured; the remaining arcs/points (which are coloured either red or green) are called the coloured arcs/points.

We construct bicoloured trails

- connecting coloured points

- and using (only) coloured arcs

by the following algorithm:

We start at some coloured point s and identify the unique coloured arc $\alpha$ incident with $s$ which is of the same colour as $s$. Then we follow the lattice path starting in $\alpha$ in the implied direction (i.e., either up/right if $s$ is a lower point, or down/left if $s$ is an upper point).

Whenever we meet another path on our way (necessarily, this path is of the other colour), we "change colour and direction", i.e., we follow this new path and change the direction (if we were moving up/right along the old path, we move down/left along the new path, and vice versa). Note that such change of colour and orientation might also occur at the very beginning: For instance, if $s$ is a green upper point, but there are (precisely) two red arcs incident with s, then we follow the red arc in the right direction.

We stop if there is no possibility to go further, i.e., if we end in another coloured point.

Figure 11 illustrates this construction (see also [6]).

Figure 11: The picture shows the bicoloured trails (as thick grey lines with rounded corners) for the example from Figure 10.

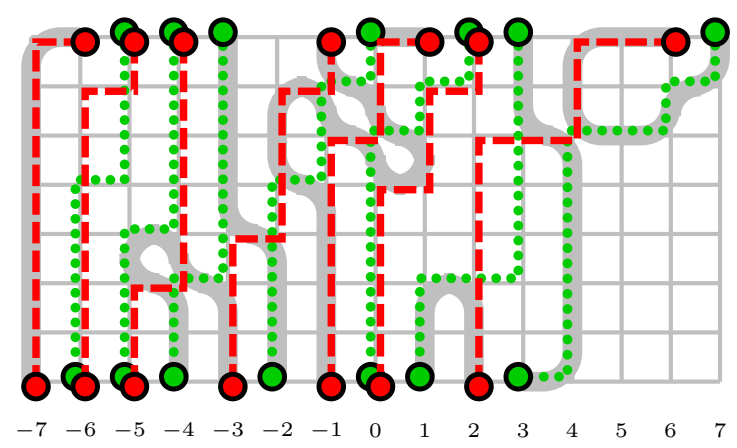


The following observations are immediate from the construction:

Observation 3 (Bicoloured trails always exist). For every coloured point s, there exists a bicoloured trail starting at $s$.

Observation 4 (Bicoloured trails can never cross). Bicoloured trails may have lattice points in common (they may intersect), but they can never cross (see Figure 12).

Figure 12: Bicoloured trails can never cross: Since bicoloured trails never use arcs coloured both red and green, a meeting point of bicoloured trails can (up to interchanging colours red and green) only occur in the situations shown below. By construction, the bicoloured trails necessarily run as indicated by the grey rounded lines: They do not cross.
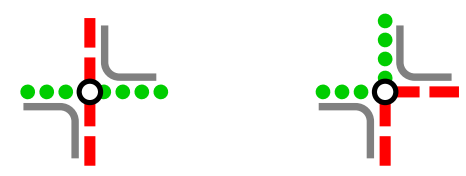

Now consider some bicoloured trail $b$ in the overlay of nonintersecting lattice paths $\left(P_{1}, P_{2}\right)$ : Changing colours (green to red and vice versa)

- of both ending points of $b$

- and of all arcs of $b$

gives a new overlay of nonintersecting lattice paths $\left(P_{1}^{\prime}, P_{2}^{\prime}\right)$ (with different lower/upper points, see Figure 13). Clearly, we have:

Observation 5 (Recolouring bicoloured trails is a weight preserving involution). The recolouring of a bicoloured trail $b$ in an overlay of nonintersecting lattice paths $\left(P_{1}, P_{2}\right)$ is an involutive operation, i.e., if we obtain the overlay $\left(P_{1}^{\prime}, P_{2}^{\prime}\right)$ by recolouring $b$ in $\left(P_{1}, P_{2}\right)$, then recolouring $b$ again in $\left(P_{1}^{\prime}, P_{2}^{\prime}\right)$ yields the original $\left(P_{1}, P_{2}\right)$. Moreover, this operation preserves the corresponding weights, i.e.,

$$
\omega\left(P_{1}\right) \cdot \omega\left(P_{2}\right)=\omega\left(P_{1}^{\prime}\right) \cdot \omega\left(P_{2}^{\prime}\right) .
$$

Note that the operation of recolouring bicoloured trails changes the colours (red/green) of coloured lower and/or upper points (which implies a change of the corresponding shapes, see Figure 13). We want to encode this change in a convenient way: Imagine that the coloured points are arranged on a circle (see Figure 14). Assign to point $s$ the radial orientation (with respect to this circle)

- inwards, if $s$ is a red upper point or a green lower point,

- outwards, if $s$ is a green upper point or a red lower point. 
Figure 13: Illustration. Recolouring two bicoloured trails (indicated by thick grey lines) from Figure 11 changes the colour of lower and/or upper points, thus changing the corresponding shape (shown below the paths).
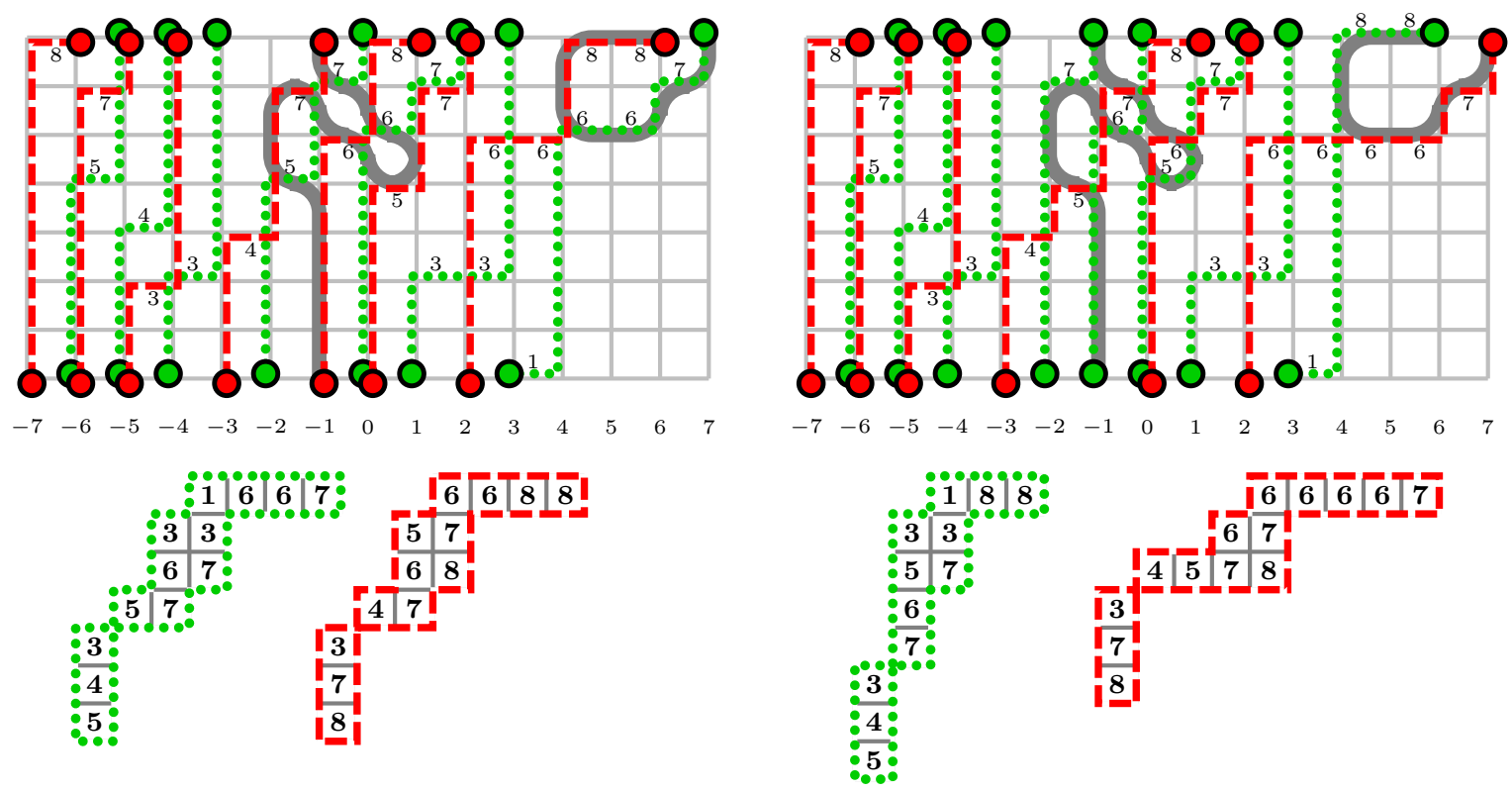

See Figure 14. From the construction of bicoloured trails, the following is immediate:

Observation 6 (Bicoloured trails connect points of different radial orientation). Bicoloured trails never connect points of the same radial orientation (i.e., two points oriented both inwards or both outwards).

Clearly, we may "forget" the colours (red or green) of the coloured points, if we remember instead their radial orientations: The situation is completely determined by

- the geometric positions of the (coloured and uncoloured) lower and upper points, we call this piece of information the configuration of $\mathbf{p}$ oints (short: $\mathbf{c p}$ ),

- together with the radial orientation of the coloured points; we call this piece of information the $\mathbf{r}$ adial orientation of $\mathbf{p}$ oints (short: rop).

Figure 15 illustrates this.

Note that for all cps, the number of coloured upper points plus twice the number of uncoloured upper points equals the number of coloured lower points plus twice the number of uncoloured lower points.

We call a rop admissible if it has the same number of inwardly/outwardly oriented points. Every admissible rop determines together with the corresponding $\mathbf{c p}$ a certain 
Figure 14: The "radial orientation" (indicated in the picture by inward/outward pointing triangles) of coloured points (shown as white circles) in their arrangement on a circle (shown as grey rectangle with rounded corners) depends on their position (upper or lower) and colour (red or green).

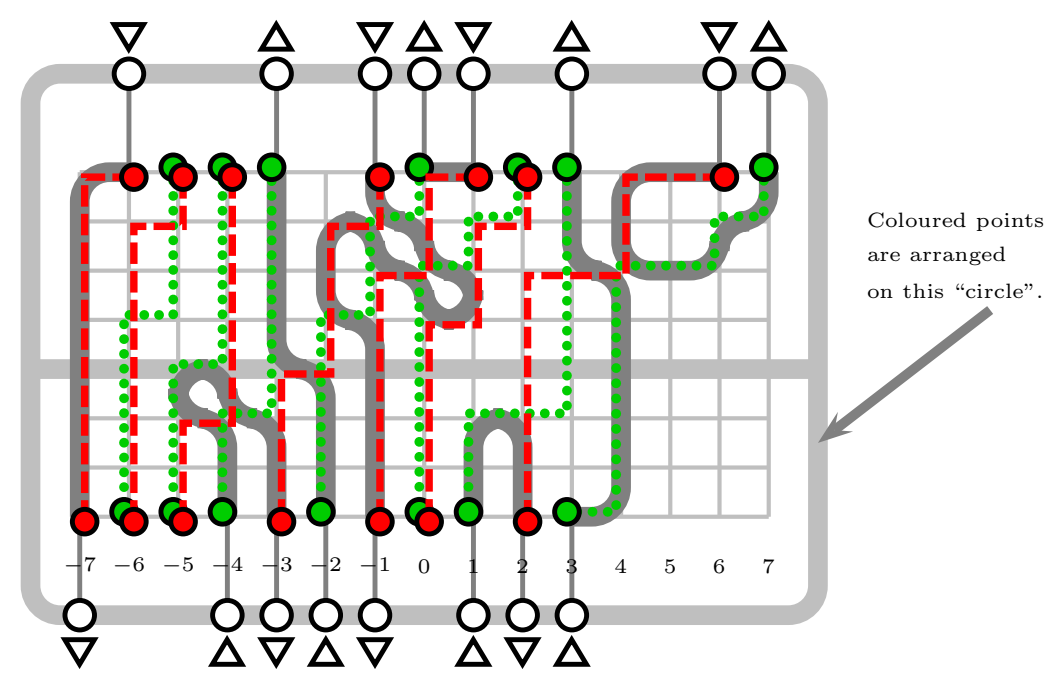

colouring (green and red) of lower/upper points, which represents a certain product of two skew Schur functions

$$
s_{\lambda / \mu} \cdot s_{\sigma / \tau}
$$

where

- $\lambda / \mu$ is determined by the uncoloured points and the green points,

- $\sigma / \tau$ is determined by the the uncoloured points and the red points.

Note that there might be no overlay of families of nonintersecting lattice paths that connect these points (if, for instance, the $i$-th green upper point lies to the left of the $i$-th green lower point; this would correspond to an $i$-th row of length $<0$ in the corresponding Ferrers diagram): In this case, the corresponding skew Schur function $s_{\lambda / \mu}$ is zero.

But if there is an overlay $\left(P_{1}, P_{2}\right)$ of families of nonintersecting lattice paths that connect the green and red points, then we may imagine that the rop corresponding to this overlay is arranged on a perfect (strictly convex) circle: Observations 3, 4 and 6 imply that if we draw a straight line connecting two points in the rop whenever the corresponding coloured points are connected by a bicoloured trail in $\left(P_{1}, P_{2}\right)$, then we will obtain a noncrossing $\mathbf{p}$ erfect $\mathbf{m}$ atching (short: $\mathbf{n p m}$ ) $\mathbf{m}$, i.e.:

- Every coloured point is incident with a straight line (edge) in $\mathbf{m}$,

- Every edge in $\mathbf{m}$ connects points of different radial orientation, 
Figure 15: The pattern of radial orientations rop (indicated by inward/outward pointing triangles) encodes the actual colours (red or green) of the coloured points in the $\mathbf{c p}$ (indicated by light grey horizontal bars in the picture; coloured/uncoloured points are shown as white/grey circles there). The bicoloured trails constitute a noncrossing perfect oriented matching (npm). The picture illustrates this for the example from Figure 14 (the edges of the npm are indicated by grey arcs).

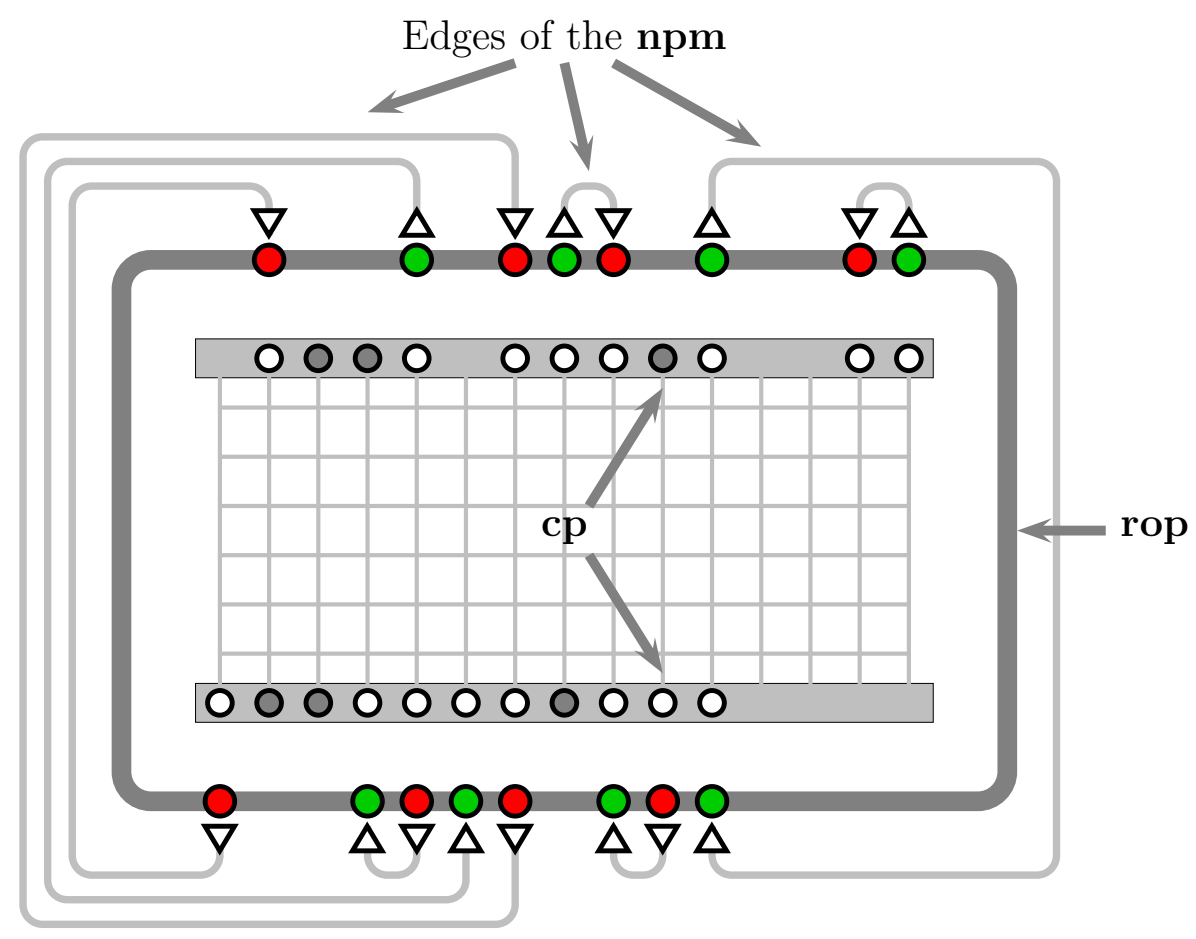

- No two edges in $\mathbf{m}$ do cross (in the geometric realization as straight lines connecting points on a circle).

In particular, this implies:

Observation 7 (Bicoloured trails span equal numbers of inward and outward directed points). Assume $p$ and $q$ are coloured points in an overlay of nonintersecting lattice paths which are connected by some bicoloured trail. Then the corresponding rop is divided in two parts by the points corresponding to $p$ and $q$ : In each of these parts, the number of points directed inwards must equal the number of points directed outwards.

Stated otherwise: Let $\mathbf{m}$ be a $\mathbf{n p m}$ for a rop $\mathbf{r}$. An arbitrary edge $e$ in $\mathbf{m}$ divides $\mathbf{r}$ into two parts, each of which must contain equal numbers of inward and outward oriented points.

We conclude our preparatory considerations with the following observation: 
Observation 8 (Admissible matchings always can be realized by overlays of nonintersecting lattice paths). For every admissible rop $\mathbf{r}$, there exists a $\mathbf{c p} \mathbf{c}$ such that every $\mathbf{n p m}$ in $\mathbf{r}$ can be realized by an overlay of nonintersecting lattice paths corresponding to $(\mathbf{c}, \mathbf{r})$ : Maybe the best way to conceive this is by looking at pictures, see Figure 16 and Figure 17.

Figure 16: Realizations of matchings, 1st part. Assume we want to realize a partial $\mathbf{n p m}$ for some subset $S$ of coloured upper points which are consecutive in the corresponding rop. In the corresponding $\mathbf{c p}$, there might be uncoloured points which are interspersed between the coloured points. As an example, consider the partial npm indicated in the upper picture. Imagine that the red and green paths are threads dangling down from the corresponding points: Note that two threads are dangling down from uncoloured points (shown as black circles), while only one thread is dangling down from coloured points (shown as white circles). Repeat the following step until there are no more coloured points: If two coloured points $p_{1}, p_{2}$ are connected by an edge $e$ of the partial $\mathbf{n p m}$, and all points between $p_{1}$ and $p_{2}$ (if any) are uncoloured, then arrange the threads dangling down from the points $p_{1}, \ldots, p_{2}$ such that they alternate in colour and tie together with knots the first and second, the third and fourth, etc., of these threads. View these knots as the new uncoloured points and simply forget the points $p_{1}, \ldots, p_{2}$ : Forgotten points are indicated by grey circles. The middle picture shows an intermediate state, where three edges of the partial npm are realized by bicoloured trails (shown as thick grey lines). The lower picture shows the final state, where there are no more coloured points, and all edges of the partial npm are realized by bicoloured trails. It is clear that the same algorithm realizes partial npms for subsets of consecutive coloured lower points.
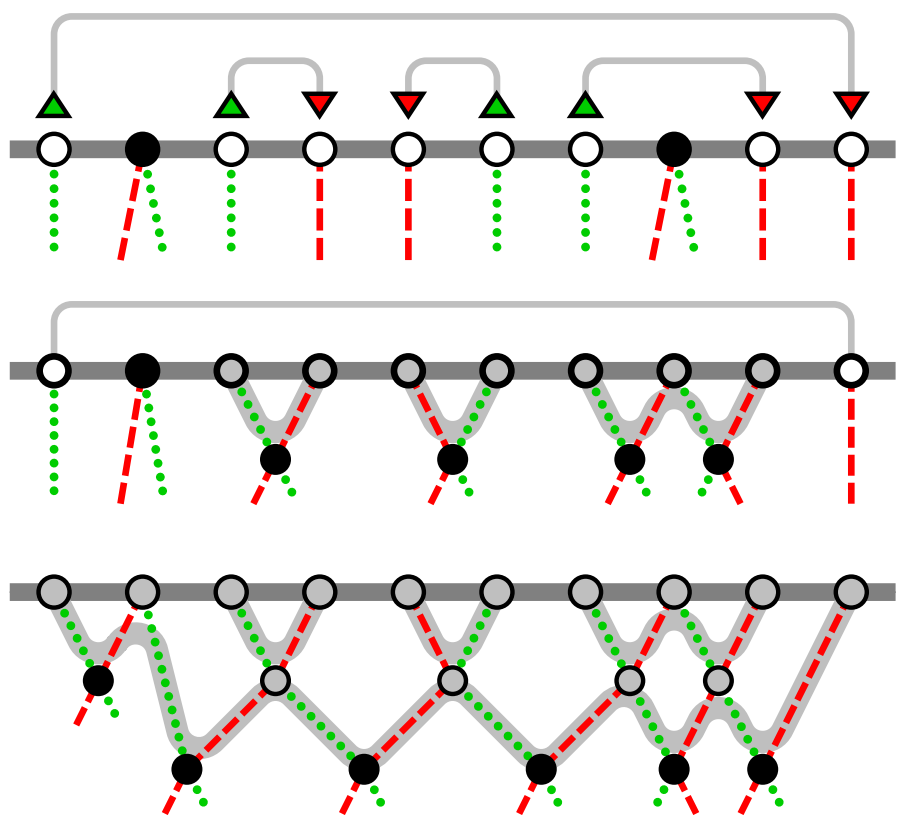
Figure 17: Realizations of matchings, 2nd part. Assume that all maximal partial npms on subsets of consecutive coloured lower or consecutive coloured upper points already have been realized, so now we have to realize edges connecting a lower point to an upper point where there is no coloured point between them (in the right half of the circular arrangement of points). The left picture indicates this situation: The edges already realized are shown as black lines. As in Figure 16, coloured points are shown as white circles, uncoloured points as black circles and forgotten points as grey circles. Obviously, by "tying together" threads of different colours (as in Figure 16) combined with "weaving together" upwards/downwards dangling threads of the same colour, we can realize the edge (shown as a grey arc) that connects these two coloured points. It is clear that this algorithm indeed gives "topological" bicoloured trails corresponding to the $\mathbf{n p m}$, and it is also clear, that the "topological" situation can be implemented "geometrically" with lattice paths for appropriately chosen shapes (with large enough distances between the upper points and lower points).
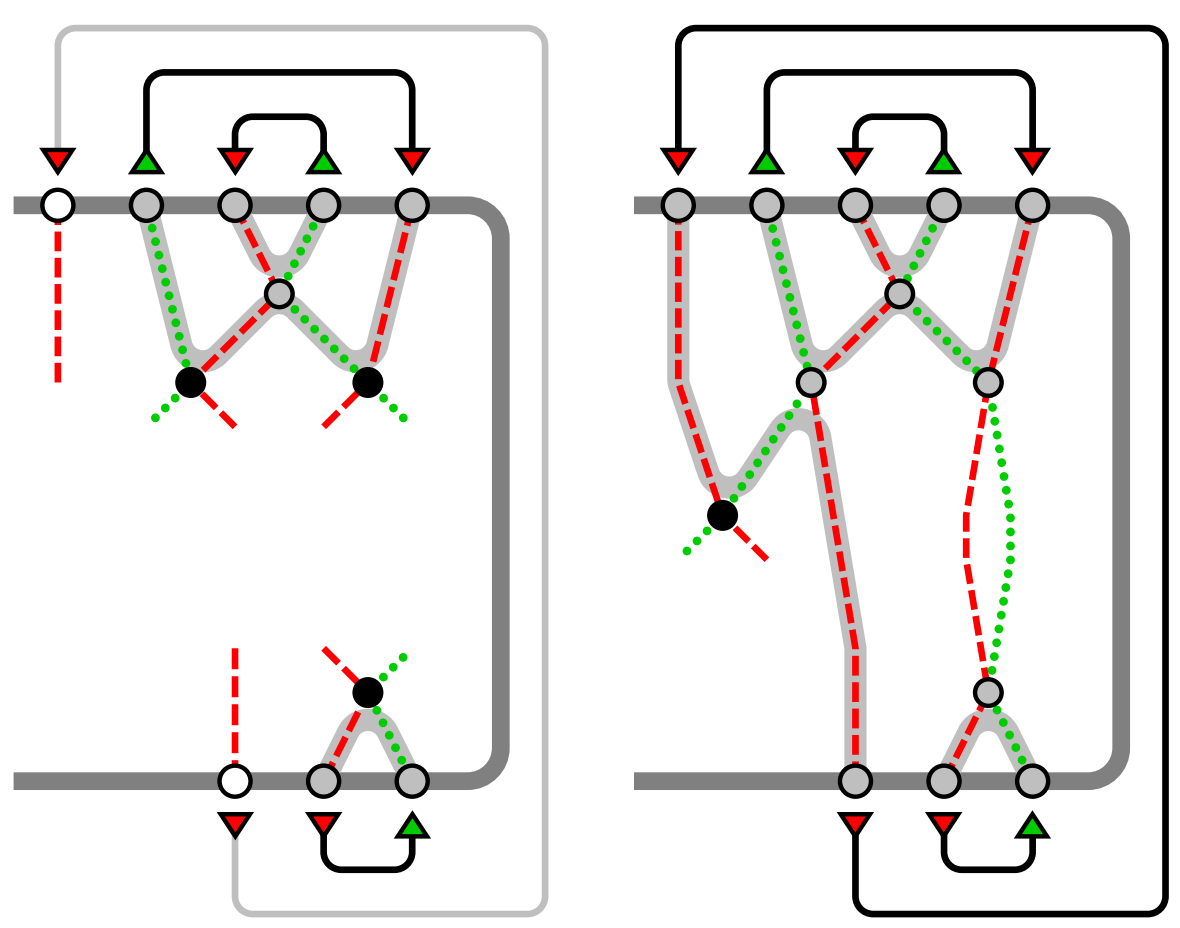

\section{Applications: Classical determinantal identities}

The simple idea for all the identities considered in the rest of this paper can be stated in an abstract manner as follows: Assume some fixed cp c.

- The set $S$ of all overlays of nonintersecting lattice paths corresponding to c and some fixed admissible rop $\mathbf{r}$ corresponds to the set of all terms in the product 
$s_{\lambda / \mu} \cdot s_{\sigma / \tau}$, where the pair of shapes $(\lambda / \mu, \sigma / \tau)$ corresponds to $(\mathbf{c}, \mathbf{r})$.

- The relation "corresponds to the same $\mathbf{n p m}$ as" is an equivalence relation on $S$. Stated otherwise, $S$ is partitioned into matching classes which are determined by the corresponding $\mathbf{n p m s} \mathbf{m}_{1}, \mathbf{m}_{2}, \ldots$ of $\mathbf{r}$ :

$$
S=S_{\mathbf{m}_{1}} \cup S_{\mathbf{m}_{2}} \cup \ldots, \text { where } S_{\mathbf{m}_{i}} \cap S_{\mathbf{m}_{j}}=\emptyset \text { for } i \neq j \text {. }
$$

- Let $\mathbf{m}_{1}$ be a fixed $\mathbf{n p m}$ in $\mathbf{r}$, and let $E\left(\mathbf{r}, \mathbf{m}_{1}\right)=\left\{e_{1}, \ldots, e_{k}\right\}$ be a fixed set of edges of $\mathbf{m}_{1}$ in $\mathbf{r}$ : Then the recolouring of the bicoloured trails $\left\{b_{1}, b_{2}, \ldots, b_{k}\right\}$ corresponding to $\left\{e_{1}, \ldots, e_{k}\right\}$ in $S_{\mathbf{m}_{1}}$ effectuates a weight-preserving involution

$$
S_{\mathbf{m}_{1}} \leftrightarrow S_{\mathbf{m}_{1}}^{\prime}
$$

where $S_{\mathbf{m}_{1}}^{\prime}$ is the set of all overlays of nonintersecting lattice paths corresponding to $\left(\mathbf{c}, \mathbf{r}^{\prime}\right)$, where $\mathbf{r}^{\prime}$ is the rop obtained from $\mathbf{r}$ by reversing the orientation of the points which are incident with the edges $\left\{e_{1}, \ldots, e_{k}\right\}$. Note that except for this reversing of orientations of points, the $\mathbf{n p m} \mathbf{m}_{1}$ appears unchanged as an $\mathbf{n p m} \mathbf{m}_{1}^{\prime}$ for $\mathbf{r}^{\prime}$ : We call this operation the reversing of the edges $E\left(\mathbf{r}, \mathbf{m}_{1}\right)$.

If we want to use this idea to obtain a Schur function identity where $\mathbf{g f}(S)$ appears on the lefthand side and $\mathbf{g f}\left(S^{\prime}\right)$ appears on the righthand side, i.e.,

$$
(\mathbf{g} \mathbf{f}(S)+\cdots)=\left(\mathbf{g} \mathbf{f}\left(S^{\prime}\right)+\cdots\right)
$$

we have to consider all triples $\left(\mathbf{c}, \mathbf{r}^{\prime}, \mathbf{m}^{\prime}\right)$, where $\mathbf{m}^{\prime}$ is an $\mathbf{n p m}$ for $\mathbf{r}^{\prime}$, i.e.,

$$
\underbrace{\left(\mathbf{g f}\left(S_{m_{1}}\right)+\mathbf{g f}\left(S_{m_{2}}\right)+\cdots\right)}_{\mathbf{g f}(S)}+\cdots=\underbrace{\left(\mathbf{g f}\left(S_{m_{1}^{\prime}}^{\prime}\right)+\mathbf{g f}\left(S_{m_{2}^{\prime}}^{\prime}\right)+\cdots\right)}_{\mathbf{g f}\left(S^{\prime}\right)}+\cdots,
$$

and have to iterate this involutive construction for all pairs $\left(\mathbf{r}^{\star}, \mathbf{m}^{\star}\right)$ of $\mathbf{r o p s} / \mathbf{n p m s}$ which arise by these operations in a consistent way. This consistency is a condition for the set of edges to be recoloured, i.e.: If the reversal of edges $E\left(\mathbf{r}^{\star}, \mathbf{m}^{\star}\right)$ leads to some set of overlays corresponding to $\left(\mathbf{r}^{\prime \prime}, \mathbf{m}^{\prime \prime}\right)$, then we must have

$$
E\left(\mathbf{r}^{\prime \prime}, \mathbf{m}^{\prime \prime}\right)=E\left(\mathbf{r}^{\star}, \mathbf{m}^{\star}\right) .
$$

We call such consistent assignment of edges (to be reversed) to pairs ( $\mathbf{r}, \mathbf{m})$ a (simple) recolouring scheme. Clearly, such recolouring scheme amounts to a weight preserving bijection

$$
S_{1} \cup S_{2} \cup \cdots \leftrightarrow S_{1}^{\prime} \cup S_{2}^{\prime} \cup \ldots,
$$

where $\left\{S_{1}, S_{2}, \ldots\right\}$ and $\left\{S_{1}^{\prime}, S_{2}^{\prime} \ldots\right\}$ are two families of sets of overlays. These two families correspond to two families

- $\left\{\left(\lambda_{1} / \mu_{1}, \sigma_{1} / \tau_{1}\right),\left(\lambda_{2} / \mu_{2}, \sigma_{2} / \tau_{2}\right), \ldots\right\}$ 
- and $\left\{\left(\lambda_{1}^{\prime} / \mu_{1}^{\prime}, \sigma_{1}^{\prime} / \tau_{1}\right),\left(\lambda_{2}^{\prime} / \mu_{2}^{\prime}, \sigma_{2}^{\prime} / \tau_{2}^{\prime}\right), \ldots\right\}$

of pairs of shapes, and the (simple) recolouring scheme thus translates to an identity involving sums of products of (skew) Schur functions

$$
s_{\lambda_{1} / \mu_{1}} \cdot s_{\sigma_{1} / \tau_{1}}+s_{\lambda_{2} / \mu_{2}} \cdot s_{\sigma_{2} / \tau_{2}}+\cdots=s_{\lambda_{1}^{\prime} / \mu_{1}^{\prime}} \cdot s_{\sigma_{1}^{\prime} / \tau_{1}^{\prime}}+s_{\lambda_{2}^{\prime} / \mu_{2}^{\prime}} \cdot s_{\sigma_{2}^{\prime} / \tau_{2}^{\prime}}+\cdots .
$$

(Figure 18 visualizes this concept.)

Figure 18: Illustration: The picture visualizes the situation where a (simple) recolouring scheme amounts to a weight preserving bijection between overlays corresponding to rops $\left\{\mathbf{r}_{1}, \mathbf{r}_{2}, \mathbf{r}_{3}\right\}$ and $\left\{\mathbf{r}_{1}^{\prime}, \mathbf{r}_{2}^{\prime}\right\}$, respectively. In the picture it is assumed that there are $6 \mathbf{n p m s}$ for these rops, which are indicated by different shades of grey (white, light grey, grey and black) and textures (slanted lines, crosshatch).

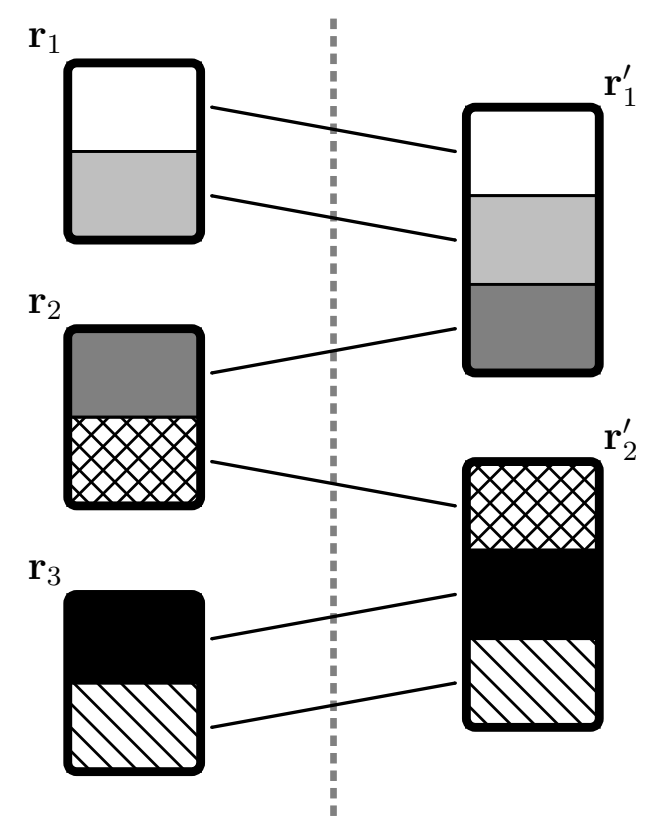

We shall give some concrete examples to make this clear.

\subsection{Dodgson's condensation, revisited and generalized}

Recall Dodgson's condensation formula (8), or rather its Schur function equivalent (11). Given our above preparations, Figure 19 contains the proof of (11)! (A simple combinatorial proof of Dodgson's condensation formula and its generalization, the Dodgson-Muiridentity, was given in [28] and in [2], respectively.)

In order to see this, we consider once again an example: Let $\lambda=\left(\lambda_{1}, \ldots, \lambda_{6}\right)=$ $(9,7,5,3,3,1)$; for convenience, we denote $\left(\lambda_{2}, \ldots, \lambda_{5}\right)$ by $\tau$. The product of Schur functions corresponding to the lefthand side of Dodgson's formula is

$$
s_{\lambda} \cdot s_{\tau}=s_{\lambda} \cdot s_{\tau+(\overline{(-1)}) /(\overline{(-1)})},
$$


which by the Lindström-Gessel-Viennot-interpretation appears as the generating function of overlays of nonintersecting lattice paths. See Figure 20, where the involutions visualized in Figure 19 are specialized to this concrete example. These weight-preserving involutions immediately imply (11), and it is obvious that the argument is valid not only for our special example, but in full generality, whence (by the reasoning following Corollary 1) we have proved (11) (and thus (8)).

Figure 19: Illustration: Graphical proof of Dodgson's condensation formula. The picture in the upper left corner shows the three rops that may appear if the bicoloured trail starting in the rightmost coloured upper point $s$ (drawn as black circle) is recoloured. Note that there is only one $\mathbf{n p m}$ for the rops in the left half, while there are two npms $\mathbf{m}_{1}, \mathbf{m}_{2}$ for the rop in the right half (the edges incident with $s$ in $\mathbf{m}_{1}$ and $\mathbf{m}_{2}$ are indicated by grey lines). The involution effectuated by the recolouring scheme is indicated by black lines connecting the corresponding rops, each of which represents a product of skew Schur functions. (The picture in the lower right corner depicts this simple recolouring scheme in the "generic" manner of Figure 18.)

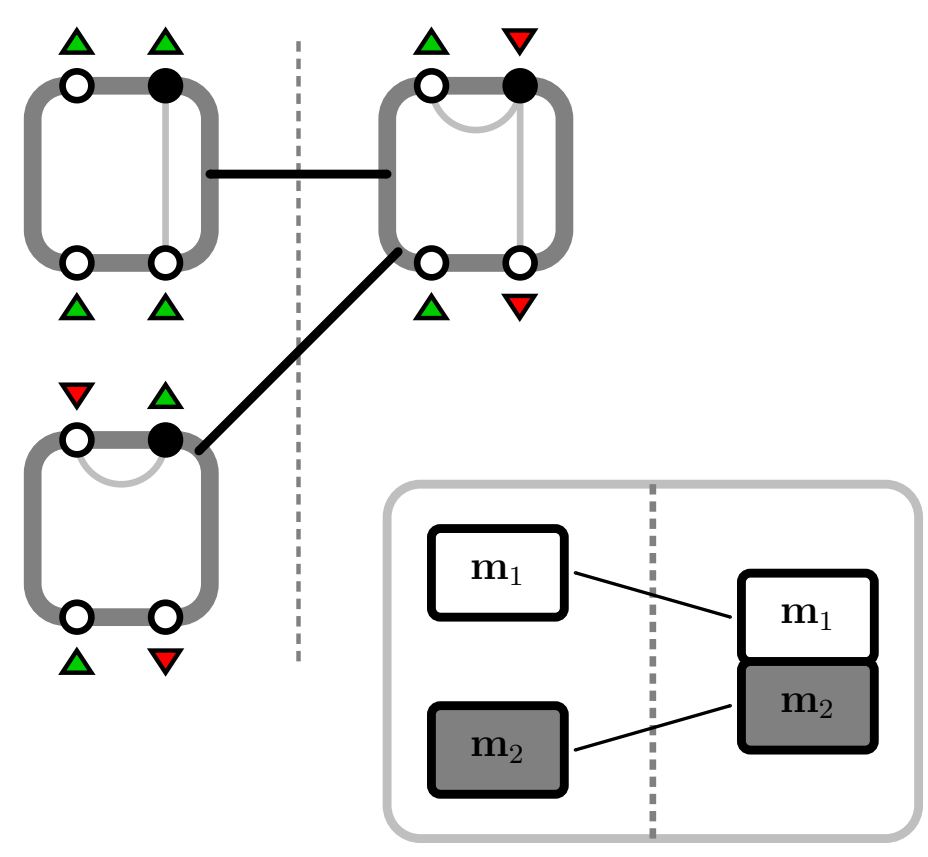

For the proof of Dodgson's condensation formula, we used a simple recolouring scheme, which we may generalize as follows: "Fix some nonempty subset $D$ of coloured points of the same orientation (i.e., all points in $S$ are either all outwards oriented or all inwards oriented). To all $\mathbf{n p m s} \mathbf{m}$ in some rop $\mathbf{r}$, assign $E(\mathbf{r}, \mathbf{m})=$ the set of edges incident with some point in $D$ ". We call this rule Dodgson's recolouring scheme: It could be restated sloppily as "always reverse all edges incident with points of $D$ ". 
Figure 20: Dodgson's condensation formula, by example.

The pictures show the positions of lower and upper points of the overlays corresponding to shapes derived from

$$
\lambda / \mu=(9,7,5,3,3,1) /(\overline{0}) \text { and } \sigma / \tau=(6,4,2,2,-1,-1, \ldots) /(\overline{(-1)}) .
$$

(For the latter we introduce the shorthand notation $\sigma / \tau=(6,4,2,2,(-1)) /(\overline{(-1)})$.) In the upper picture, all coloured points are green, and the bicoloured trail $b$ starting in the rightmost upper point $s=(8, N)$ must have the rightmost lower point $(-1,1)$ as its other ending point. Recolouring $b$ leads to the middle picture. Here, the bicoloured trail starting in $s$ has two possibilities: Its other ending point might be $(-1,1)$ again, but also the leftmost upper point $(-5, N)$ is possible. For the latter case, recolouring the bicoloured trail connecting $s$ and $(-5, N)$ leads to the lower picture. Now, as in the upper picture, the bicoloured trail starting in $s$ must have $(-5, N)$ again as its other ending point. By the above reasoning, this shows immediately the following instance of (11):

$$
s_{\lambda / \mu} \cdot s_{\sigma / \tau}+s_{\lambda^{\prime \prime} / \mu^{\prime \prime}} \cdot s_{\sigma^{\prime \prime} / \tau^{\prime \prime}}=s_{\lambda^{\prime} / \mu^{\prime}} \cdot s_{\sigma^{\prime} / \tau^{\prime}}
$$

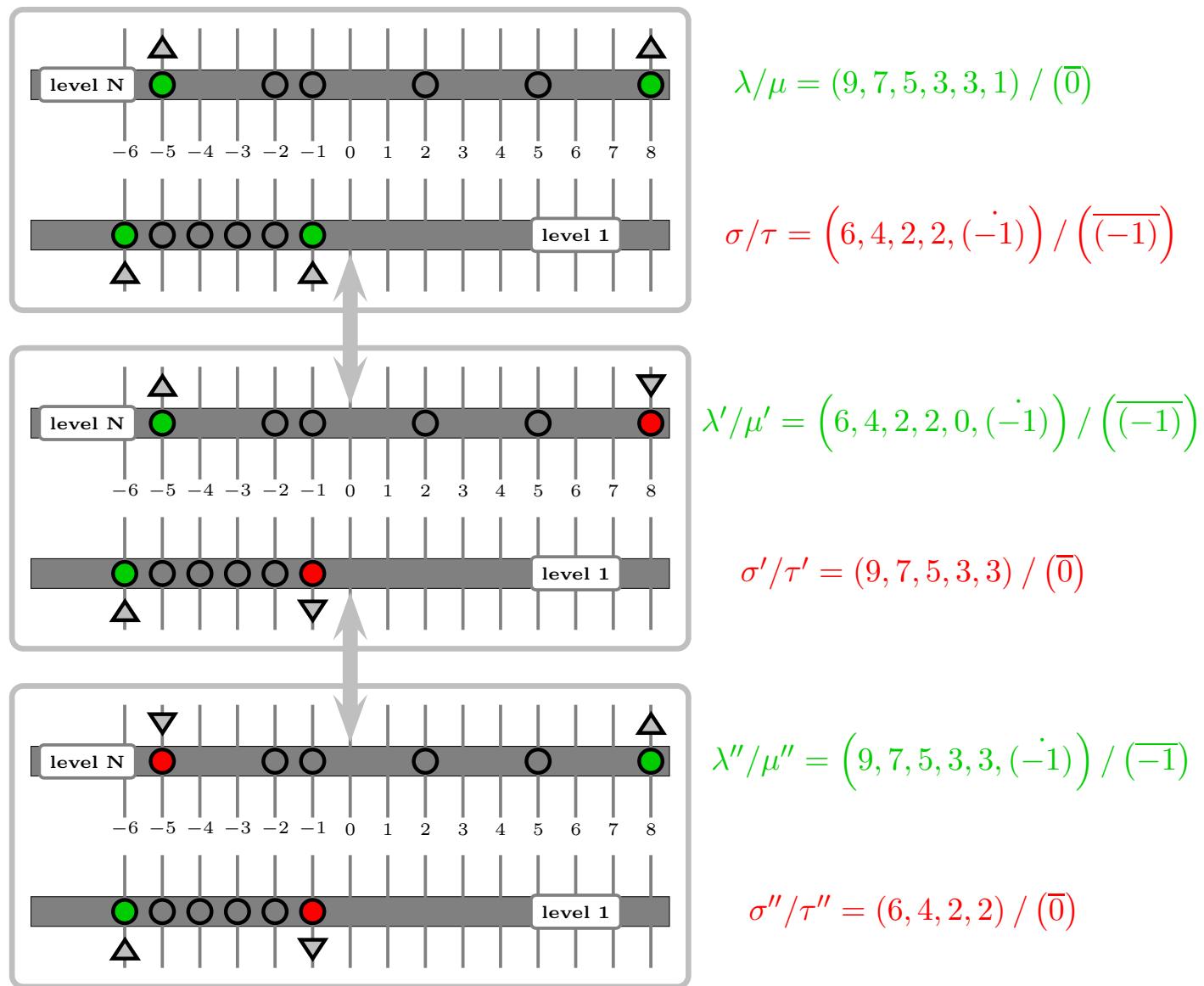


The following Lemma and its proof are a reformulation of [6, Lemma 15]):

Lemma 1. Except for degenerate cases, Dodgson's recolouring scheme always yields a Schur function identity.

Proof. Clearly, Dodson's recolouring scheme consistently determines the set of edges to be reversed for every $\mathbf{n p m}$. The following reasoning is merely a clarification of the statement:

Let $\lambda / \mu$ and $\sigma / \tau$ be two skew shapes and consider the $\mathbf{c p} \mathbf{c}$ which corresponds to $(\lambda / \mu, \sigma / \tau)$. Without loss of generality, let $D$ be a nonempty subset of inwards oriented points in $\mathbf{c}$.

Let $V$ be the set of all admissible rops for c where all points of $D$ have the same orientation. Consider the graph $G$ with vertex set $V$, where two vertices $v_{1}, v_{2}$ are connected by an edge if and only if there exists a $\mathbf{n p m} \mathbf{~} \mathbf{m}$ for $v_{1}$ such that $v_{2}$ is obtained by applying Dodgson's recolouring scheme (i.e., reverse all edges in $\mathbf{m}$ which are incident with a point of $D$ ). Clearly, this graph $G$ is bipartite: $V=I \cup O$, where $I$ is the subset of $V$ with all points in $D$ oriented inwards and $O$ is the subset of $V$ with all points in $D$ oriented outwards, and there is no edge connecting two vertices of $I$ or two vertices of $O$ (see again Figure 19, where the bipartite structure is indicated by a dashed line separating the left and right half of the picture).

Assume that graph $G$ has a connected component $Z$ with at least 2 vertices (if there is no such component, we call this a degenerate case). Then we have the following identity for skew Schur functions:

$$
\sum_{(\lambda / \mu, \sigma / \tau) \in Z_{I}} s_{\lambda / \mu} \cdot s_{\sigma / \tau}=\sum_{\left(\lambda^{\prime} / \mu^{\prime}, \sigma^{\prime} / \tau^{\prime}\right) \in Z_{O}} s_{\lambda^{\prime} / \mu^{\prime}} \cdot s_{\sigma^{\prime} / \tau^{\prime}}
$$

where $Z_{O}$ and $Z_{I}$ denote the sets of pairs of skew shapes corresponding to $(\mathbf{c}, x)$ for $x \in O$ and $x \in I$, respectively.

We illustrate Lemma 1 by the following generalization of Dodgson's condensation, which might be new. Consider the rop with $k$ upper and $k$ lower points $(k>1)$ which are all green, and always recolour the bicoloured trail starting in the rightmost upper point ( $k=2$ corresponds to Dodgson's condensation, see Figure 19; the cases $k=3$ and $k=4$ are depicted in Figures 21 and 22, respectively).

Theorem 2. Let a be an $(m+k) \times(m+k)$-matrix, and let $1 \leqslant i_{1}<i_{2}<\cdots<i_{k} \leqslant m+k$ and $1 \leqslant j_{1}<j_{2}<\cdots<j_{k} \leqslant m+k$ be (the indices of) $k$ fixed rows and $k$ fixed columns, respectively, of a. Denote the sets of these (indices of) rows and columns by $R$ and $C$, respectively.

Let $E:=\left\{j_{2}, j_{4}, \ldots, j_{2 \cdot\lfloor k / 2\rfloor}\right\}$ and $O:=\left\{i_{1}, i_{3}, \ldots, i_{2 \cdot\lceil k / 2\rceil-1}\right\}$ be the sets of odd fixed row indices and of even fixed column indices, respectively. Then we have:

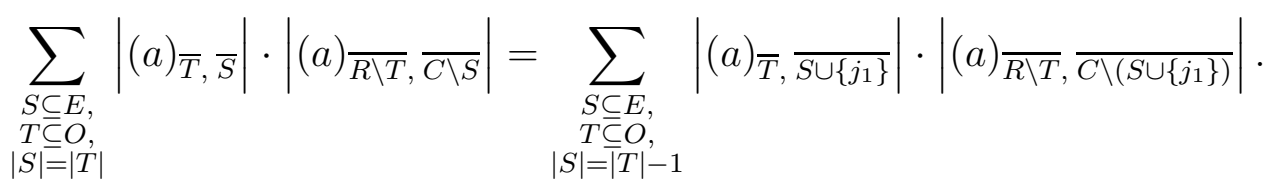


Figure 21: Dodgson's recolouring scheme, where the set $D$ consists only of the rightmost coloured upper point (drawn as black circle), applied to 3 coloured upper/lower points.

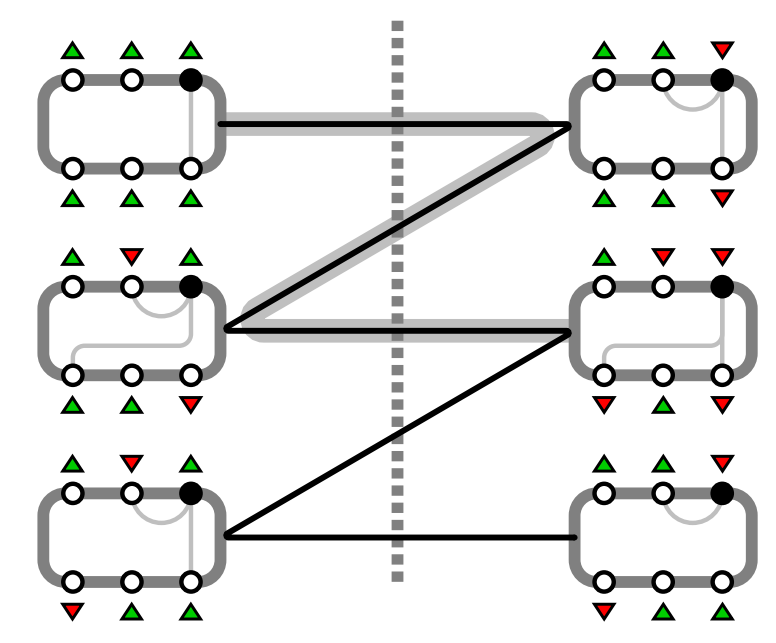

Proof. We shall prove the Schur function identity equivalent to (23): Note that the corresponding $\mathbf{c p}$ shows $m+k$ lower points, of which $k$ are coloured, and $m+k$ upper points, of which $k$ are coloured. As always, we concentrate on the coloured points: Denote the upper coloured points by $t_{1}, t_{2}, \ldots, t_{k}$, and the lower coloured points by $s_{1}, s_{2}, \ldots, s_{k}$ (counted from the right, as always). Consider the rop where all coloured points are green (see the uppermost configuration in the left parts of Figures 21 and 22): This rop corresponds to the summand for $S=T=\emptyset$ in the left-hand side of (23). Clearly, the other end of a bicoloured trail starting in the rightmost upper point $t_{1}$ must either be in the set $O:=\left\{s_{1}, s_{3}, \ldots, s_{2 \cdot\lceil k / 2\rceil-1}\right\}$ or in the set $E:=\left\{t_{2}, t_{4}, \ldots, t_{2 \cdot\lfloor k / 2\rfloor}\right\}$. Now observe that it is possible to recolour points $s_{1}, t_{2}, s_{3}, t_{4}, \ldots$ (in this order; these recolouring steps are indicated by thick grey lines in Figures 21 and 22) until we obtain the rop where all points in $E \cup O$ are coloured red. (Note that in this rop, $t_{1}$ is green if $k$ is even, otherwise $t_{1}$ is red.) Now it is easy to see that for an arbitrary choice of subsets $S, T$, where $S \subseteq E \cup\left\{t_{1}\right\}$, $T \subseteq O$ and $|S|=|T|$, by the recolouring of red upper and lower points in the appropriate order, we can obtain the situation where the set of red points is precisely the union $S \cup T$ : This translates to the assertion.

As an example, we state the determinantal identity corresponding to Figure $22(k=4)$ for the special case where $a$ is a $4 \times 4$-matrix (i.e., $m=0$ ):

$$
\begin{aligned}
|a|=\left|(a)_{1,1}\right| \cdot\left|(a)_{\overline{1}, \overline{1}}\right|+\left|(a)_{1,3,1,2}\right| \cdot\left|(a)_{\overline{1,3}, \overline{1,2}}\right| & +\left|(a)_{1,3,1,4}\right| \cdot\left|(a)_{\overline{1,3}, \overline{1,4}}\right|+\left|(a)_{3,1}\right| \cdot\left|(a)_{\overline{3}, \overline{1}}\right| \\
-\left|(a)_{1,2}\right| \cdot\left|(a)_{\overline{1}, \overline{2}}\right|-\left|(a)_{1,3,2,4}\right| \cdot\left|(a)_{\overline{1,3}, \overline{2,4}}\right|-\left|(a)_{3,2}\right| \cdot\left|(a)_{\overline{3}, \overline{2}}\right| & -\left|(a)_{1,4}\right| \cdot\left|(a)_{\overline{1}, \overline{4}}\right|-\left|(a)_{3,4}\right| \cdot\left|(a)_{\overline{3}, \overline{4}}\right| .
\end{aligned}
$$


Figure 22: Dodgson's recolouring scheme, where the set $D$ consists only of the rightmost coloured upper point (drawn as black circle), applied to 4 coloured upper/lower points.

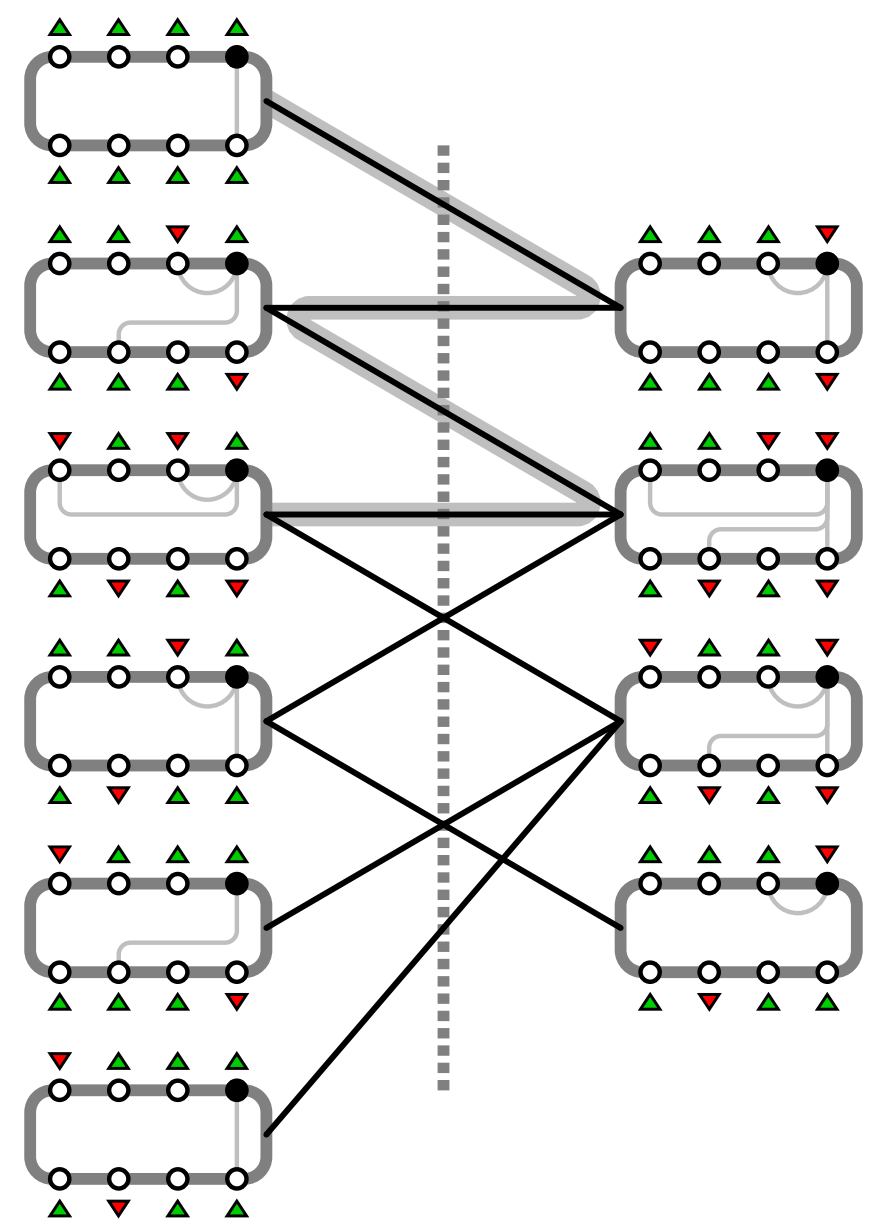

\section{2 (Generalized) Plcker relations}

There is a particularly simple special case of Lemma 1 (this is a reformulation of $[6$, Lemma 16]):

Corollary 2. Let $\mathbf{r}$ be the rop for the pair of shapes $(\lambda / \mu, \sigma / \tau)$, and assume that the orientation of the points in $\mathbf{r}$ is alternating (with respect to their circular arrangement). Consider the set of all rops which arise by applying Dodgson's recolouring scheme (for some fixed nonempty set of points of the same orientation) to $\mathbf{r}$ and denote the corresponding set of pairs of skew shapes by $Q$.

Then we have:

$$
s_{\lambda / \mu} \cdot s_{\sigma / \tau}=\sum_{\left(\lambda^{\prime} / \mu^{\prime}, \sigma^{\prime} / \tau^{\prime}\right) \in Q} s_{\lambda^{\prime} / \mu^{\prime}} \cdot s_{\sigma^{\prime} / \tau^{\prime}}
$$


Proof. Consider the rop $\mathbf{r}^{\prime}$ corresponding to some Schur function product $s_{\lambda^{\prime} / \mu^{\prime}} \cdot s_{\sigma^{\prime} / \tau^{\prime}}$ from the right hand side of (24): By the combination of Observations 6 and 4, it is clear that re-applying Dodgson's recolouring scheme always yields the rop $\mathbf{r}$ corresponding to the Schur function product $s_{\lambda / \mu} \cdot s_{\sigma / \tau}$.

We will use this Corollary for a proof of the Plcker relations (also known as GrassmannPlücker syzygies, see [24], or as Sylvester's Theorem, see [21, section 137]). In addition to notation $[m]:=\{1,2, \ldots, m\}$ we introduce the notation

$$
([m]+n):=\{n+1, n+2, \ldots, n+m\} .
$$

Moreover, for finite ordered sets $X \subseteq S$ and $Y$ with $Y \cap S=\emptyset,|Y|=|X|$, we introduce the notation $\left(\left.S\right|_{\leftarrow Y} ^{X \rightarrow}\right)$ for the set $S$ where the elements of $X$ are replaced by the elements of $Y$ in the same order, i.e., if ordered sets $X$ and $Y$ are given as $X=\left(x_{1}, x_{2}, \ldots\right)$ and $Y=\left(y_{1}, y_{2}, \ldots\right)$, respectively, and $S$ is given as

$$
S=\left(s_{1}, \ldots, s_{\left(k_{1}-1\right)}, x_{1}, s_{\left(k_{1}+1\right)}, \ldots, s_{\left(k_{2}-1\right)}, x_{2}, s_{\left(k_{2}+1\right)}, \ldots\right),
$$

then ordered set $\left(\left.S\right|_{\leftarrow Y} ^{X \rightarrow}\right)$ is given as

$$
\left(\left.S\right|_{\leftarrow Y} ^{X \rightarrow}\right)=\left(s_{1}, \ldots, s_{\left(k_{1}-1\right)}, y_{1}, s_{\left(k_{1}+1\right)}, \ldots, s_{\left(k_{2}-1\right)}, y_{2}, s_{\left(k_{2}+1\right)}, \ldots\right) .
$$

Theorem 3 (Plcker relations). Let $a=\left(a_{i, j}\right)_{(i, j) \in[m] \times[2 m]}$ be a $m \times 2 \cdot m$ matrix. Consider some fixed set $R \subseteq[m]$. Then we have

$$
\left|(a)_{[m],[m]}\right| \cdot\left|(a)_{[m],([m]+m)}\right|=\sum_{\substack{S \subseteq([m]+m),|S|=|R|}}\left|(a)_{[m],\left(\left.[m]\right|_{\leftarrow S} ^{R \rightarrow}\right)}\right| \cdot\left|(a)_{[m],\left(([2 m] \backslash[m])||_{\leftarrow R}^{S \rightarrow}\right)}\right| \cdot
$$

Proof. We shall prove a Schur function identity which is equivalent to (25): Consider

$$
\lambda=((2 \cdot m) \cdot(m-1),(2 \cdot m-2) \cdot(m-1), \ldots, 4 \cdot(n-1), 2 \cdot(n-1))
$$

and

$$
\sigma=((2 \cdot m-1) \cdot(m-1),(2 \cdot m-3) \cdot(m-1), \ldots, 3 \cdot(n-1),(n-1))
$$

and assume that matrix $(a)_{[m],[m]}=\mathbf{h}_{\lambda}$ and matrix $(a)_{([m]+m),[m]}=\mathbf{h}_{\sigma}$. It is clear that the rop corresponding to the pair of shapes $(\lambda /(\overline{0}), \sigma /(\overline{0}))$ has only upper coloured points which alternate in orientation, see the configurations in the left part of Figure 23. Corollary 2 immediately translates to (the Schur function equivalent of) (25); see Figure 23 for an illustration.

Note that the proof (which basically is contained in Figure 23!) implies an obvious generalization: Of course, there might be uncoloured points in the cps associated to the rops shown in Figure 23! Such uncoloured points amount to a slightly more general Plücker-like identity involving a common minor in the products of determinants (similar to Theorem 2). (In [10, (3.3)], this identity is stated by describing the situation with certain operations of Ferrers diagrams and proved by using the Plücker relations; the connection of this identity to the more general statements presented here was already explained ad hoc in [5].) 
Figure 23: Illustration of the Plücker relation (25) for $m=3$ : The left part shows three copies of the rop considered in the proof of Theorem 3 with different npms; the right part shows the effect of recolouring the fixed points which are indicated by black circles. Note that there are five possible npms in this situation: For the upper and lower pictures, there are two npms which yield the same recolouring; these two npms are shown in the left and right halves of the pictures, respectively. In the middle picture, there is only one npm (which is the same in the left half and in the right half).

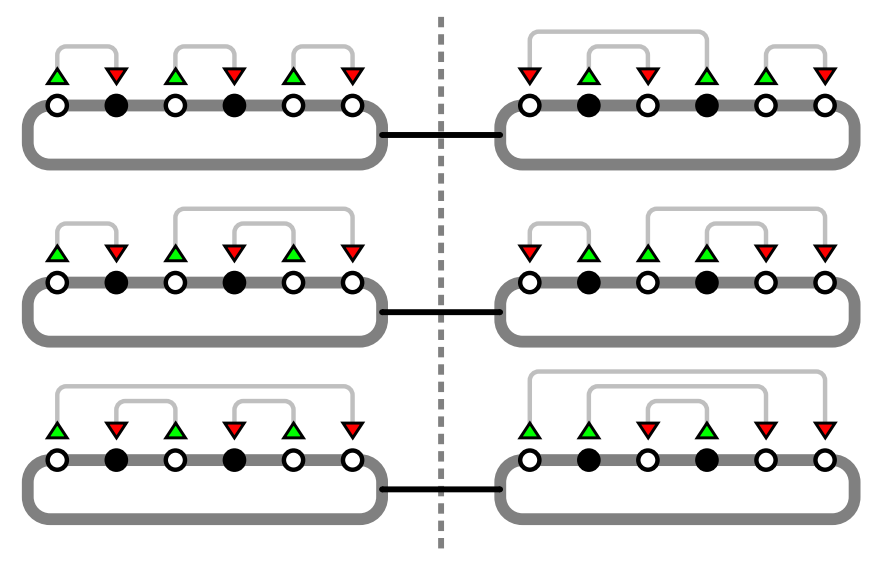

Theorem 4. Let a be an $(m+k) \times(m+2 \cdot k)$-matrix, and let $1 \leqslant j_{1}<j_{2}<\cdots<$ $j_{2 k} \leqslant m+2 \cdot k$ be (the indices of) $2 \cdot k$ fixed columns of $a$, and set $A:=\left\{j_{1}, \ldots, j_{k}\right\}$ and $O:=\left\{j_{k+1}, \ldots, j_{2 \cdot k}\right\}$. Let $A^{\prime}:=[m+2 \cdot k] \backslash A$ and $O^{\prime}:=[m+2 \cdot k] \backslash O$. Consider some fixed set $R \subseteq A$. Then we have:

$$
\left|(a)_{[m+k], A^{\prime}}\right|\left|(a)_{[m+k], O^{\prime}}\right|=\sum_{\substack{S \subseteq O,|S|=|R|}}\left|(a)_{[m+k],\left(A^{\prime} \mid \stackrel{R}{\leftarrow S}\right)}\right| \cdot\left|(a)_{[m+k]},\left(\left.O^{\prime}\right|_{\leftarrow R} ^{S \rightarrow}\right)\right| .
$$

\subsection{Generalized recolouring scheme}

For Laplace's Theorem (stated here as Theorem 5), we need a slight generalization of simple recolouring schemes. Observe that it is not necessary for our purposes that a recolouring scheme constitutes a bijective mapping between matching classes (as visualized in Figure 18); it suffices that it establishes a "bipartite structure" in the following sense:

- Let $\mathbf{m}$ be a fixed $\mathbf{n p m}$ for $(\mathbf{c}, \mathbf{r})$ (where $\mathbf{r}$ is a rop for $\mathbf{c p} \mathbf{c}$ ), and let

$$
\mathcal{E}(\mathbf{r}, \mathbf{m})=\left\{E_{1}(\mathbf{r}, \mathbf{m}), \ldots, E_{k}(\mathbf{r}, \mathbf{m})\right\}
$$

be a fixed family of sets of edges of $\mathbf{m}$ in $\mathbf{r}$ : Then the reversing of edges in $E_{1}, \ldots, E_{k}$ 
amounts to a $k$-valued relation

$$
\begin{aligned}
& S_{\mathbf{m}} \leftrightarrow S_{\mathbf{m}}^{1} \text { (weight-preserving bijection), } \\
& \ldots \\
& S_{\mathbf{m}} \leftrightarrow S_{\mathbf{m}}^{k} \text { (weight-preserving bijection), }
\end{aligned}
$$

where

- $S_{\mathbf{m}}$ is the set of all overlays of nonintersecting lattice paths corresponding to $(\mathbf{c}, \mathbf{r}, \mathbf{m})$,

- $S_{\mathbf{m}}^{i}$ is the set of all overlays of nonintersecting lattice paths corresponding to $\left(\mathbf{c}, \mathbf{r}_{i}^{\prime}, \mathbf{m}\right)$, where $\mathbf{r}_{i}^{\prime}$ is the rop obtained from $\mathbf{r}$ by reversing the edges $E_{i}$.

- Assume that we can assign to each pair $(\mathbf{r}, \mathbf{m})$ a family of sets of edges consistently, i.e., such that $\mathcal{E}(\mathbf{r}, \mathbf{m}) \cap \mathcal{E}\left(\mathbf{r}^{\prime}, \mathbf{m}\right) \supseteq\{E\}$ if $\mathbf{r}^{\prime}$ is obtained by $\mathbf{r}$ by reversing the edges E.

- Assume further that the graph $G$

- with vertex set equal to all matching classes $S_{\mathbf{m}}^{\prime}$,

- where the edges are given by the weight-preserving bijections $S_{\mathbf{m}}^{\prime} \leftrightarrow S_{\mathbf{m}}^{\prime \prime}$ just described

is bipartite such that for every connected component of $G$ the bipartition classes are of equal size.

Then we call such assignment of families of edges a (generalized) recolouring scheme. It is clear, that a recolouring scheme corresponds to a Schur function identity. This idea is visualized in Figure 24.

\subsubsection{Laplace expansion}

Assume we are given some rop $\mathbf{r}$ with at least $m$ lower points $s_{1}, \ldots, s_{m}$. Consider the following recolouring scheme: Fix lower points $S:=\left\{s_{i_{1}}, \ldots, s_{i_{k}}\right\}$ in $\mathbf{r}(k \leqslant m)$. For every npm $\mathbf{m}$, reverse

- one of the edges of $\mathbf{m}$ connecting two consecutive coloured upper points (we call such edge an upper handle), if there is one,

- else all the edges of $\mathbf{m}$ starting at a point of $S$.

(I.e., the family $\mathcal{E}(\mathbf{r}, \mathbf{m})$ of edges assigned to $(\mathbf{r}, \mathbf{m})$ is either the family of sets (singletons), where each set contains an upper handle, or it consists of the single set of edges which are incident with points in $S$.) A moment's thought shows that this is a recolouring scheme in the sense just described: We call this the Laplace recolouring scheme. 
Figure 24: Illustration: The picture visualizes the situation where a recolouring scheme amounts to a weight preserving bijection between overlays corresponding to rops $\left\{\mathbf{r}_{1}, \mathbf{r}_{2}, \mathbf{r}_{3}\right\}$ and $\left\{\mathbf{r}_{1}^{\prime}, \mathbf{r}_{2}^{\prime}\right\}$, respectively. In the picture it is assumed that there are only 4 npms for these rops, which are indicated by different shades of grey (white, light grey, grey and black). The crucial point is that the recolouring scheme assigns every npm to a "bipartite substructure" with bipartition classes of the same size (the edges of these "bipartite substructures" are shown as thin black lines). The simplest case of such "bipartite substructure" appears for the black $\mathbf{n p m} \mathbf{m}_{\text {black }}$ : There is a mapping taking $\left(\mathbf{r}_{3}, \mathbf{m}_{\text {black }}\right)$ to $\left(\mathbf{r}_{2}^{\prime}, \mathbf{m}_{\text {black }}\right)$ and vice versa. However, this is not the only possibility: Note that, for example, every white $\mathbf{n p m} \mathbf{m}_{\text {white }}$ is related to two instances of $\mathbf{m}_{\text {white }}$ : The corresponding "bipartite substructure" has bipartitions classes $\left\{\left(\mathbf{r}_{1}, \mathbf{m}_{\text {white }}\right),\left(\mathbf{r}_{3}, \mathbf{m}_{\text {white }}\right)\right\}$ and $\left\{\left(\mathbf{r}_{1}^{\prime}, \mathbf{m}_{\text {white }}\right),\left(\mathbf{r}_{2}^{\prime}, \mathbf{m}_{\text {white }}\right)\right\}$ (both of size 2$)$.

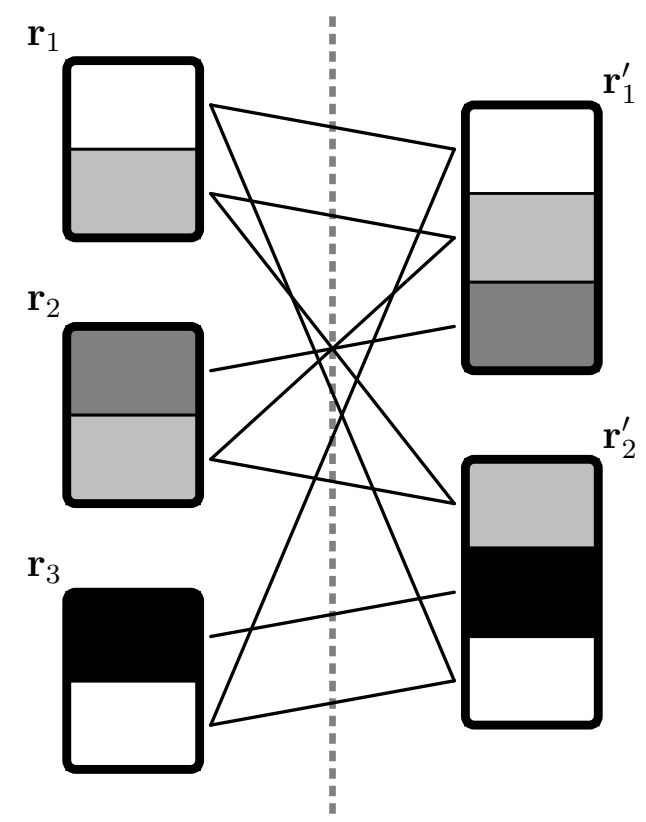

Applying this scheme for $S=\{j\}$ (i.e., $k=1$ ) to the cp with $m$ upper and $m$ lower points, $m>1$, all of which are coloured green, implies the Laplace expansion of the $m \times m$-determinant by its $j$-th column

$$
|a|=\sum_{i=1}^{m}(-1)^{i-j} \cdot\left|a_{i, j}\right| \cdot\left|(a)_{\bar{i}, \bar{j}}\right| .
$$

As for Dodgson's condensation, Figure 25 contains the proof: The concrete example presented there illustrates the case $m=4, j=1$, and generalizes to a general Schur function identity, which implies (27). (Observe that this gives, in fact, a simple recolouring scheme, since there can be at most one upper handle in this case.) 
Figure 25: The proof of the Laplace expansion of the $4 \times 4$-determinant.

The proof is contained in the pictures below. The upper point $s$ of the bicoloured trail to be recoloured is indicated by a coloured circle, and the possible connections by bicoloured trails are indicated by grey arcs. Note that for some partition $\lambda=\left(\lambda_{1}, \ldots, \lambda_{4}\right)(m=4)$, the involutions (indicated in the pictures by thick black lines) imply that the generating function of the left half,

$$
\left.\left.s_{\lambda} \cdot s_{(0)}+s_{(\lambda+(1\lfloor m\rceil)}\right)^{\overline{2}} \cdot s_{\left(\lambda_{2}-1\right)}+s_{(\lambda+(1\lfloor m\rceil)}\right)^{\overline{4}} \cdot s_{\left(\lambda_{4}-3\right)},
$$

is equal to the generating function of the right half,

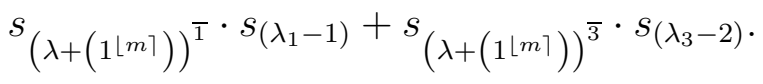

By the connections between minors of $\mathbf{h}_{\sigma / \tau}$ and operations on partitions $\sigma$ and $\tau$ (which were explained before Corollary 1), this means $\left|\mathbf{h}_{\lambda}\right|=\left|\left(\mathbf{h}_{\lambda}\right)_{\overline{1}, \overline{1}}\right| \cdot\left(\mathbf{h}_{\lambda}\right)_{1,1}-\left|\left(\mathbf{h}_{\lambda}\right)_{\overline{2}, \overline{1}}\right| \cdot\left(\mathbf{h}_{\lambda}\right)_{2,1}+$ $\left|\left(\mathbf{h}_{\lambda}\right)_{\overline{3}, \overline{1}}\right| \cdot\left(\mathbf{h}_{\lambda}\right)_{3,1}-\left|\left(\mathbf{h}_{\lambda}\right)_{\overline{4}, \overline{1}}\right| \cdot\left(\mathbf{h}_{\lambda}\right)_{4,1}$, which implies the Laplace expansion by the first column.

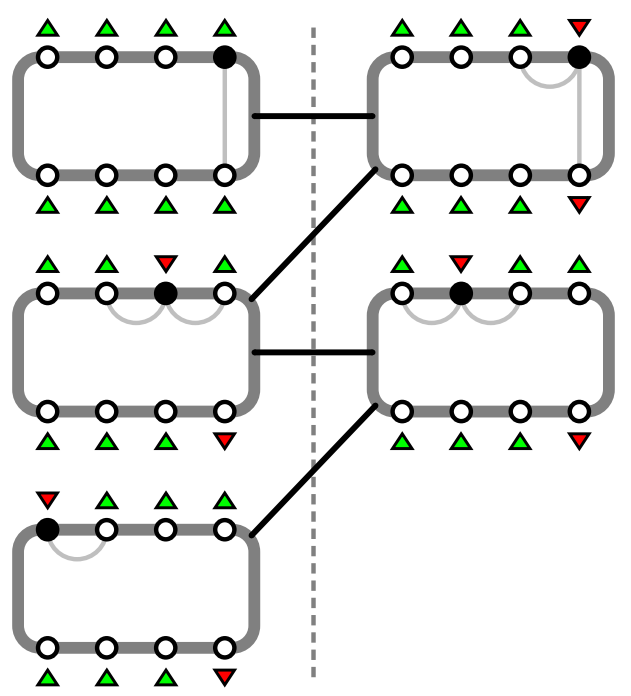

\subsubsection{Laplace's Theorem}

For the generalization of Laplace's expansion (see [21, section 93]), we introduce the following notation: Let $X=\left\{x_{1}, \ldots, x_{m}\right\} \subset \mathbb{Z}$ be an ordered set, and let $S=\left\{x_{i_{1}}, \ldots, x_{i_{k}}\right\}$, $k \leqslant m$, be a subset. In this situation, we define

$$
\Sigma_{S \subseteq X}:=\sum_{j=1}^{k} i_{j} .
$$


Theorem 5 (Laplace's Theorem). Let $a=\left(a_{i, j}\right)_{(i, j) \in[m] \times[m]}$ be an $m \times m$-matrix. Consider some fixed set $I \subseteq[m]$. Then we have

$$
|a|=\sum_{\substack{J \subseteq[m],|\bar{J}|=|I|}}(-1)^{\Sigma_{I \subseteq[m]}+\Sigma_{J \subseteq[m]}} \cdot\left|(a)_{I, J}\right| \cdot\left|(a)_{\bar{I}, \bar{J} \mid}\right| .
$$

Figure 26: Illustration of Laplace's Theorem, for $m=5$ and $I=\{2,4\}$ : The lower points corresponding to $I$ are indicated by black circles. The $\mathbf{n p m}$ shown in the pictures has two upper handles (edges connecting neighbouring upper points); handles that have been "reversed" (i.e., their endpoints were recoloured) are drawn in black. The important point is that the two bipartition classes (corresponding to the left and the right half of the picture) are of the same size; see also Figure 24 where this is situation appears in a more "abstract" way.

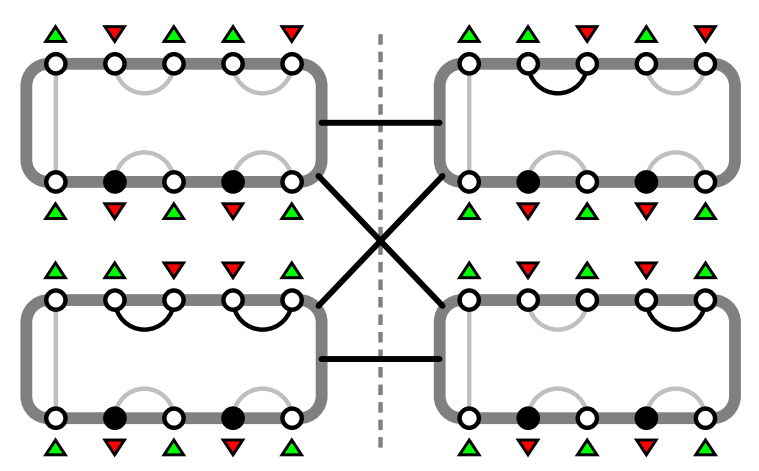

Proof. As always, we consider the equivalent Schur function identity: The rop corresponding to the left-hand side of (28) consists of

- $m$ upper points, which are outward oriented,

- and $m$ lower points, which are inward oriented

(stated otherwise: All $2 \cdot m$ coloured points are green). We may assume $0<|I|<m$ (otherwise, there is nothing to prove), i.e., $I$ corresponds to $k$ lower points $s_{i_{1}}, \ldots, s_{i_{k}}$, $0<k<m$.

Now we apply the Laplace recolouring scheme. Note that a $\mathbf{n p m} \mathbf{m}$ always contains an upper handle if it contains an edge connecting two upper points. If there is more than one upper handle, the recolouring scheme is not simple, i.e., it does not describe a mapping, but a multi-valued relation on pairs $(\mathbf{r}, \mathbf{m})$, where $\mathbf{r}$ is a rop and $\mathbf{m}$ is a $\mathbf{n p m}$ in $\mathbf{r}$ : It is easy to see that nevertheless a bipartite substructure occurs (see Figure 24): Starting with some arbitrary but fixed pair $(\mathbf{r}, \mathbf{m})$, by the Laplace recolouring scheme all the pairs $\left(\mathbf{r}^{\prime \prime}, \mathbf{m}\right)$ which are obtained by reversing an even number of upper handles, 
end up in the same bipartition class as $(\mathbf{r}, \mathbf{m})$ : Clearly, these are of the same number as all the pairs $\left(\mathbf{r}^{\prime}, \mathbf{m}\right)$ which are obtained by reversing an odd number of upper handles (constituting the other bipartition class). See Figure 26 for an illustration.

Note that the same proof also works for cps which contain uncoloured points: This amounts to a slightly more general statement (see [21, section 148])

Theorem 6. Let a be an $(m+k) \times(m+k)$-matrix, and let $1 \leqslant i_{1}<i_{2}<\cdots<i_{m} \leqslant m+k$ and $1 \leqslant j_{1}<j_{2}<\cdots<j_{m} \leqslant m+k$ be (the indices of) $k$ fixed rows and $k$ fixed columns of a. Denote the set of these (indices of) rows and columns by $R$ and $C$, respectively. Consider some fixed set $I \subseteq R$. Then we have:

$$
|a| \cdot\left|(a)_{\bar{R}, \bar{C}}\right|=\sum_{\substack{J \subseteq C,|J|=|I|}}(-1)^{\Sigma_{I \subseteq R}+\Sigma_{J \subseteq C}} \cdot(a)_{\overline{R \backslash I}}, \overline{C \backslash J} \cdot(a)_{\bar{I}, \bar{J}} .
$$

\subsection{Turnbull's identity}

Let $M$ be an $n \times(n \cdot p)$-matrix, which we view as "vector of columns $c_{k}$ ":

$$
M=\left(c_{1}, c_{2}, \ldots, c_{n \cdot p}\right) .
$$

Consider an arrangement of the $n \cdot p$ symbols $c_{i}$ in a $p \times n$-tableau $T$. Clearly, every such arrangement may be viewed as a permutation $\sigma$ of the symbols in the "canonical arrangement"

$$
\left(\begin{array}{cccc}
c_{1} & c_{p+1} & \cdots & c_{(n-1) \cdot p+1} \\
c_{2} & c_{p+2} & \cdots & c_{(n-1) \cdot p+2} \\
\vdots & \vdots & \vdots & \vdots \\
c_{p} & c_{2 \cdot p} & \cdots & c_{n \cdot p}
\end{array}\right)
$$

We view $T$ as an "encoding" for the product of the minors given by $T$ 's rows, multiplied by the sign of the corresponding permutation $\sigma$. For example, for $n=3, p=2$ we have

$$
\left(\begin{array}{lll}
c_{2} & c_{3} & c_{5} \\
c_{1} & c_{4} & c_{6}
\end{array}\right)=-\operatorname{det}\left(c_{2}, c_{3}, c_{5}\right) \times \operatorname{det}\left(c_{1}, c_{4}, c_{6}\right) .
$$

By abuse of notation, we again call this signed product a tableau and denote it by $T$.

Observe that a permutation of elements from the same row in $T$ does not change the value of the corresponding signed product of minors. In this sense, we consider two tableaux differing only by such "permutations inside rows" as equivalent: When we consider sets of tableaux in the following, it goes without saying that these tableaux are assumed to be pairwise non-equivalent.

Let $T$ be a $p \times n$ tableau, and let $A$ and $B$ be two disjoint subsets of the entries of $T$. Imagine that the elements of $A$ are indicated by boxes, and the elements of $B$ are indicated by circles. Then we will denote by $T^{\square}$ or $T^{\bigcirc}$ the sum over all (pairwise nonequivalent) tableaux which can be obtained from $T$ by permuting the elements of $A$ or $B$, respectively. For example, consider the following $2 \times 3$-tableau with $A=\{a, b, e, f\}$ and $B=\emptyset$ : 


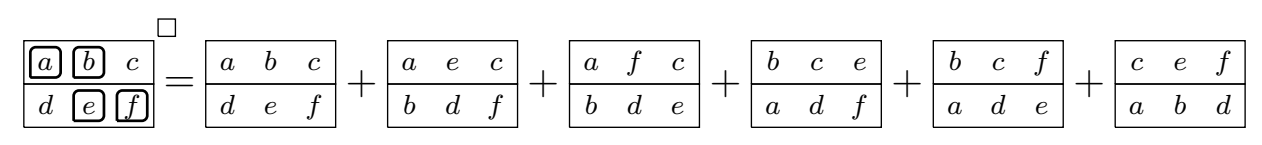

In 1909, Turnbull stated the following generic identity [17, Proposition 1.2.2]; see also [26], [27, p. 48] and [20, V, p.51]):

Theorem 7 (Turnbull's identity). Let $T$ be a $p \times n$-tableau, let $A$ be the subset of "boxed" entries of $T$, and set $k:=|A|$.

Denote by $R$ the set of entries from the first row in $T$, and define the subset of "circled" elements $B:=R \backslash A$.

If $k>n$, then $T^{\square} \equiv 0$.

If $k \leqslant n$, we may construct another tableau $T_{B}$ by exchanging all the elements of $A \backslash R$ with elements of $B$. Set $m:=|A \backslash R|$. Then we have

$$
T^{\square} \equiv(-1)^{m} \cdot T_{B}^{\bigcirc} .
$$

Of course, we want to present a proof of (31) in terms of recolouring bicoloured trails. So from now on, we assume that for every tableaux $T^{\prime}$, which is obtained by permuting entries of the "original" tableau $T$, the entries in each row of $T^{\prime}$ appear in the lexicographic order determined by $T$ (see (30)):

$$
T_{i, j}<T_{k, l}: \Longleftrightarrow j<l \text { or }(j=l \text { and } i<k) .
$$

We may identify the $k$-th entry $T_{i, j}$ (in this order) of $T^{\prime}$ with the $k$-th (coloured) endpoint $e$ of lattice paths (ordered from right to left), where the colour of $e$ is determined by the row $l$ in which $T_{i, j}$ appears in $T^{\prime}$.

We illustrate this by the following example for Turnbull's identity:

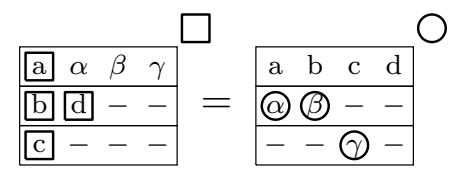

I.e., we consider a $3 \times 4$-tableau $T$ with $A=\{a, b, c, d\}$ and $B=\{\alpha, \beta, \gamma\}$ (we do not introduce notations for the remaining entries of $T$ since they are never involved in any permutation of entries): Figure 27 shows the tableaux involved in this identity.

From the pictures in Figure 27 it is not obvious how to view Turnbull's identity directly as a recolouring scheme: In fact, our proof takes the following detour.

Lemma 2. Let $T$ be a $p \times n$-tableau, let $A \neq \emptyset$ be the subset of "boxed" entries of $T$, and set $k:=|A|$.

Denote by $R$ the set of entries from the first row in $T$, and define the subset of "circled" elements $B:=R \backslash A$. Set $m:=|A \backslash R|$.

Consider the set $T^{A \cup B}$ of tableaux which are obtained from $T$ by permuting marked (boxed or circled) elements, i.e., elements from $A \cup B$. Denote by $T_{\mathrm{o}}^{A \cup B}$ the set of all tableaux in $T^{A \cup B}$ with 
- exactly $k$ circled entries

- and exactly $(m-k)$ boxed entries

in the rows $>1$. Then we have the following $m$ identities:

$$
\sum_{j=0}^{m}\left(\begin{array}{l}
j \\
k
\end{array}\right) \operatorname{gf}\left(T_{\circ \equiv k}^{A \cup B}\right)=0 \text { for } 0 \leqslant k<m .
$$

Proof. View the determinant corresponding to the $i$-th row of $T$ as the generating function of nonintersecting lattice paths with starting points

$$
(-1,1),(-2,1), \cdots,(-n+1,1), \ldots,(-n, 1)
$$

and with ending points

$$
(n \cdot p-i, N),((n-1) \cdot p-i, N), \cdots,((2 \cdot p)-i, N),((1 \cdot p)-i, N) .
$$

Consider the set $T^{A \cup B}$ of all tableaux which are obtained from $T$ by rearranging the marked (boxed or circled) elements from $A \cup B$. Assume that the order (32) for the entries of $T$ is inherited for every row in every $T^{\prime} \in T^{A \cup B}$ : Then each tableau $T^{\prime} \in T^{A \cup B}$ can be described by the same starting and ending points, where only the colours change: For each such $T^{\prime}$, assign colour $c_{i}$ to the ending points whose corresponding entry appears in row $i$ in $T^{\prime}$; denote these points by $c_{i}\left(T^{\prime}\right)$.

For some arbitrary subset $F \subseteq B$ of circled entries, denote by $T_{\circ \equiv F}^{A \cup B}$ the family of all tableaux in $T^{A \cup B}$ where the set of circled entries in rows $>1$ is precisely $F$, and denote by $T_{\circ \supseteq F}^{A \cup B}$ the family of all tableaux in $T^{A \cup B}$ where the set of circled entries in rows $>1$ contains $F$.

We claim that for an arbitrary subset $F \subseteq B$ with $0 \leqslant|F|=k<m$ there holds:

$$
\mathbf{g f}\left(T_{\circ \supseteq F}^{A \cup B}\right) \equiv 0 .
$$

This is easily established by observing that the recolouring scheme

Choose a marked ending point $e \in\left(A \cup B \backslash c_{1}\left(T^{\prime}\right)\right) \backslash F$; assume $e$ has colour $c_{i}, i>1$. Recolour the bicoloured trail starting in $e$ in the overlay of lattice paths of colours $c_{1}$ and $c_{i}$.

yields a weight-preserving and sign-reversing "involutive bipartite structure" on $T_{F}^{A \cup B}$ : Note that there is such marked ending point $e$ by assumption, since

$$
|F|<m=\left|A \cup B \backslash c_{1}\left(T^{\prime}\right)\right| .
$$

Thus we obtain by summing and elementary counting

$$
0=\sum_{\substack{F \subseteq B,|F|=k}} \operatorname{gf}\left(T_{\circ \supseteq F}^{A \cup B}\right)=\sum_{\substack{M \subseteq B,|M|=j \geqslant k}}\left(\begin{array}{l}
j \\
k
\end{array}\right) \operatorname{gf}\left(T_{\circ \equiv M}^{A \cup B}\right)=\sum_{j=0}^{m}\left(\begin{array}{l}
j \\
k
\end{array}\right) \operatorname{gf}\left(T_{\circ \equiv j}^{A \cup B}\right) .
$$


Figure 27: Graphical representations of the tableaux corresponding to (33). In the picture, the tableaux are shown together with the corresponding coloured endpoints: Endpoints corresponding to the set $A$ are boxed, endpoints corresponding to the set $B$ are circled. The colour of the endpoints indicates the row they belong to: Black endpoints belong to the first row, red endpoints belong to the second row, and green endpoints (shown as dotted points in the pictures) belong to the third row. The tableaux with positive sign appear on the left side, and the tableaux with negative sign appear on the right side.

The tableaux corresponding to the left-hand side of (33):

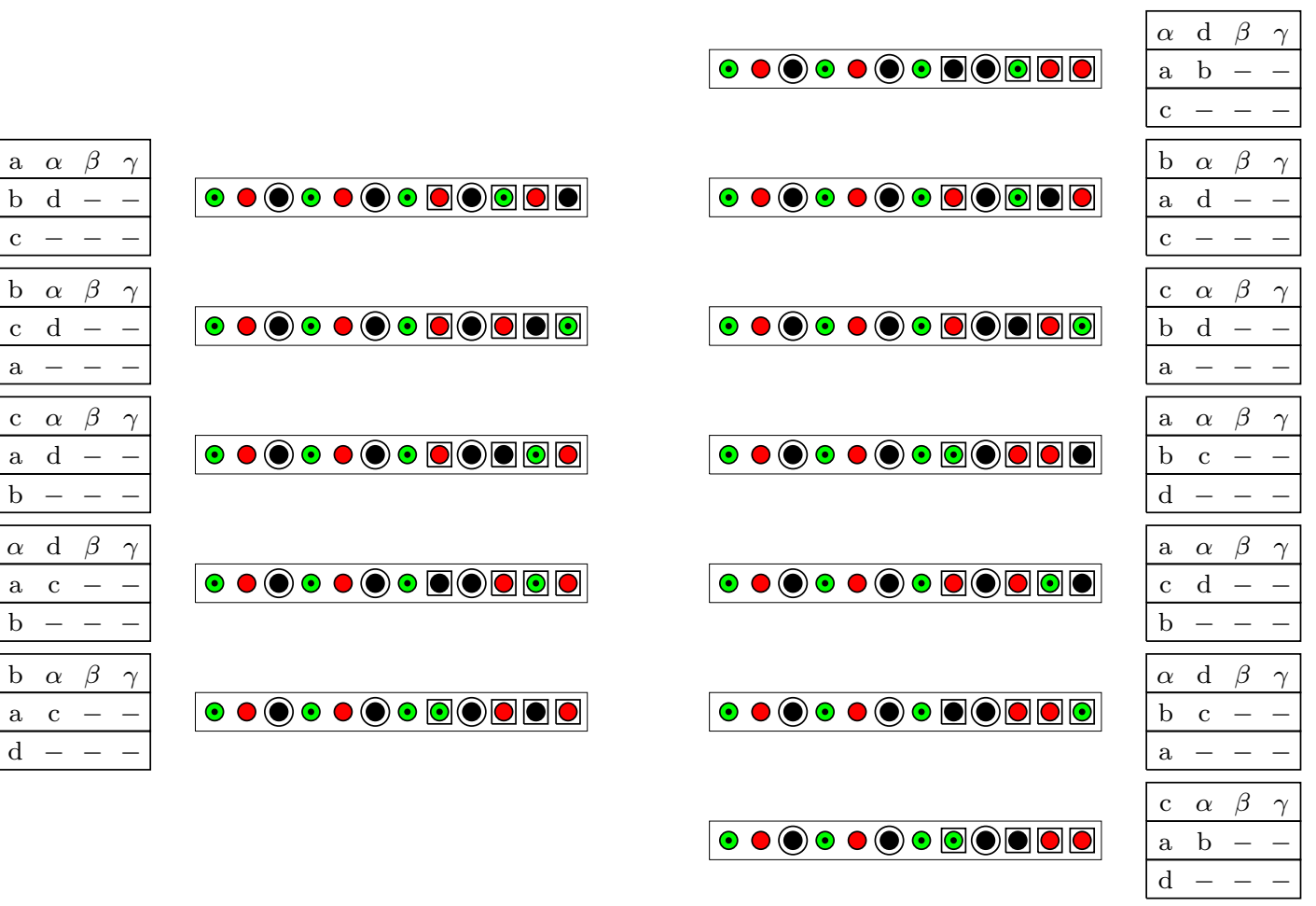

The tableaux corresponding to the right-hand side of (33):

\begin{tabular}{|cccc|}
\hline $\mathrm{a}$ & $\mathrm{b}$ & $\mathrm{c}$ & $\mathrm{d}$ \\
\hline$\alpha$ & $\beta$ & - & - \\
\hline- & - & $\gamma$ & - \\
\hline
\end{tabular}

$\odot \circ \bigcirc \odot ० \odot \odot 0 \bigcirc 000$

$\odot ० \odot \odot ० \bigcirc \odot \bigcirc \bigcirc 000$

$\odot \circ \bigcirc \odot \bigcirc \bigcirc \odot \odot 000$

\begin{tabular}{|lllll|}
\hline $\mathrm{a}$ & $\mathrm{b}$ & $\mathrm{c}$ & $\mathrm{d}$ \\
\hline$\alpha$ & - & $\gamma$ & - \\
\hline- & $\beta$ & - & - \\
\hline $\mathrm{a}$ & $\mathrm{b}$ & $\mathrm{c}$ & $\mathrm{d}$ \\
\hline$\beta$ & - & $\gamma$ & - \\
\hline$\alpha$ & - & - & - \\
\hline
\end{tabular} 
Corollary 3. Under the assumptions and with the definitions from Lemma 2 we have

$$
T_{\mathrm{o} \equiv 0}^{A \cup B}=(-1)^{m} T_{\circ}^{A \cup B},
$$

which amounts to Turnbull's identity.

Proof. The alternating sum of equations (34) gives

$$
\begin{aligned}
0 & =\sum_{i=0}^{m-1}(-1)^{i} \sum_{j=0}^{m}\left(\begin{array}{l}
j \\
i
\end{array}\right) T_{\circ \equiv j}^{A \cup B} \\
& =\sum_{j=0}^{m} T_{\circ}^{A \cup B}\left(\sum_{i=0}^{m-1}(-1)^{i}\left(\begin{array}{l}
j \\
i
\end{array}\right)\right) \\
& =T_{\circ}^{A \cup B}-(-1)^{m} T_{\mathrm{o} \equiv m}^{A \cup B} .
\end{aligned}
$$

(Note that $T_{\circ \equiv m}^{A \cup B}=0$ if $k>n$.)

\section{References}

[1] M. Aigner. Lattice paths and determinants. In Helmut Alt, editor, Computational Discrete Mathematics, Lecture Notes in Comput. Sci., pages 1-12. Springer-Verlag New York, Inc., 2001.

[2] A. Berliner and R. A. Brualdi. A combinatorial proof of the Dodgson/Muir determinantal identity. Int. J. Inf. Syst. Sci., 4(1):1-7, 2008.

[3] D. Bressoud. Proofs and Confirmations: The Story of the Alternating Sign Matrix Conjecture. Cambridge University Press, New York, 1999.

[4] C.L. Dodgson. Condensation of determinants, being a new and brief method for computing their arithmetic values. Proceed. Roy. Soc. London, 15:150-155, 1866.

[5] M. Fulmek. Bijective proofs for Schur function identities. arXiv:0909.5334v1 [math.CO], 2009.

[6] M. Fulmek and M. Kleber. Bijective proofs for Schur function identities which imply Dodgson's condensation formula and Plücker relations. Electron. J. Combin., 8(1):Research Paper 16, 22 pp. (electronic), 2001.

[7] I.M. Gessel and X. Viennot. Binomial determinants, paths, and hook length formulae. Adv. in Math., 58:300-321, 1985.

[8] I.M. Gessel and X. Viennot. Determinants, paths, and plane partitions. preprint, 1989.

[9] I.P. Goulden. Quadratic forms of skew Schur functions. European J. of Combinatorics, 9:161-168, 1988.

[10] Dimitri Gurevich, Pavel Pyatov, and Pavel Saponov. Bilinear identities on Schur symmetric functions. Journal of Nonlinear Mathematical Physics, 17(Supplementary Issue 1):31-48, 2010. also available as arXiv:0907.4292v2 [math.CO]. 
[11] C.G. Jacobi. De formatione et proprietatibus determinantium. Journal für Reine und Angewandte Mathematik, 22:285-318, 1841.

[12] C.G. Jacobi. De functionibus alternantibus earumque divisione per productum e differentiis elementorum conflatum. Crelle's Journal, 22:360-371, 1841.

[13] S. Karlin. Coincident probabilities and applications in combinatorics. J. Appl. Probab., 25A:185-200, 1988.

[14] A.N. Kirillov. Completeness of states of the generalized Heisenberg magnet. J. Soviet Math., 1987.

[15] M. Kleber. Plücker relations on Schur functions. Journal of Algebraic Combinatorics, 13:199-211, 2001.

[16] J. Lagrange. Solutions analytiques de quelques problèmes sur les pyramides triangulaires. Nouv. Mém. de l'Acad. Roy. Sci. Bel.-Let. Berlin, pages 149-176, 1773.

[17] B. Leclerc. On identities satisfied by minors of a matrix. Adv. Math., 100(1):101-132, 1993.

[18] B. Lindström. On the vector representation of induced matroids. Bull. London Math. Soc., 5:85-90, 1973.

[19] I.G. Macdonald. Symmetric Functions and Hall Polynomials. Oxford Science Publications, 2nd edition, 1995.

[20] T. Muir. The Theory of Determinants in the historical order of development. MacMillan and Co., Limited, 1906.

[21] T. Muir. A Treatise on the Theory of Determinants. Longmans, Green and Co., 1933.

[22] B.E. Sagan. The Symmetric Group. Springer, 2nd edition, 2000.

[23] Issai Schur. Über eine Klasse von Matrizen, die sich einer gegebenen Matrix zuordnen lassen. PhD thesis, Berlin, 1901.

[24] B. Sturmfels. Algorithms in Invariant Theory. Texts and Monographs in Symbolic Computation. Springer-Verlag, Wien, 1993.

[25] N. Trudi. Intorno un determinante piu generale di quelle che suol dirsi determinante delle radici di una equazione, ed alle funzioni simmetriche complete di questo radici. Rend. Accad. Sci. Fis. Mat. Napoli, 3:121-134, 1864.

[26] H.W. Turnbull. The irreducible concomitants of two quadratics in $n$ variables. Trans. Cambridge Philos. Soc., 21:197-240, 1909.

[27] H.W. Turnbull. The Theory of Determinants, Matrices and Invariants. Dover Publications, Inc., 3rd edition, 1960.

[28] D. Zeilberger. Dodgson's determinant-evaluation rule proved by TWO-TIMING MEN and WOMEN. Electr. J. Comb., 1997. 